\title{
Oral health condition of the Brazilian adolescents and its influence on dental diversity patterns for human identification
}

\author{
Deitos, Alexandre Raphael *; Azevedo, Alana de Cássia Silva *; Michel-Crosato, Edgard *; \\ Biazevic, Maria Gabriela Haye * \\ * School of Dentistry, Universidade de São Paulo (FOUSP), Brazil
}

\section{ABSTRACT}

Introduction: Forensic dentistry makes possible the identification of a great number of individuals. The combination of the anatomical variability of the teeth with the several types of dental treatment creates numerous dental patterns.

Aim: The aim of the study was to verify if the improvement in the oral health condition of the Brazilian adolescents would interfere in the analysis of the dental diversity patterns and its potential use for human identification in forensic sciences.

Materials and methods: The use of the clinical dental condition records, available in the database from the last two Brazilian National Oral Health Surveys (2003-2010), enabled the assessment of dental patterns.

Results: The national and regional conditional diversity values calculated for complete and partial dentition (common situation in a mass disaster event) -0.911 to $0.997, \mathrm{P}>0.05$ are similar to the diversity patterns values of mitochondrial DNA.

Conclusion: The improvement in the oral health condition of Brazilian adolescents did not interfere with the potential use of dental diversity patterns as an objective method of human identification. However, it is necessary to develop other methodologies to potentiate the use of forensic dentistry, since oral health conditions change over time.

Deitos AR, Azvedo A, Michel-Crosato E, Biazevic M. Oral health condition of the Brazilian adolescents and its influence on dental diversity patterns

for human identification. South Eur J Orthod Dentofac Res. 2015;2(2)35-43.

Submitted: October 05, 2014; Revised: March 17, 2015; Published: September 30, 2015

\section{INTRODUCTION}

Forensic dentistry, especially the comparison of dental characteristics and dental treatments, is the key to identification of a great number of individuals. It is one of the most effective methods for identifying humans after disasters because the teeth and their treatments often survive environmental conditions that destroy other identifying features. ${ }^{1}$ Complete and detail - rich antemortem and postmortem data make such identifications more plausible. ${ }^{2}$

The combination of the anatomical variability of the teeth among individuals with the several types of dental treatment

\section{Corresponding Author:}

Maria Gabriela Haye Biazevic

Departamento de Odontologia Social, School of Dentistry,

Universidade de Sáo Paulo (FOUSP),

Avenida Professor Lineu Prestes, 2227 05508-000, Sáo Paulo-SP, Brazil

e-mail: biazevic@usp.br performed creates numerous dental patterns. ${ }^{3}$ Adams (2003) ${ }^{4}$ showed the necessity of exploring the dental diversity patterns (missing, filled, and higid teeth) for forensic identification when the more frequently used statistical methods are inaccurate or inappropriate. He used statistical arguments based on the number of possible combinations of dental characteristics to suggest that an individual's dental health pattern should be more frequently used for identification due to its singularity. Forensic comparisons of non-radiographic dental evidence antemortem and postmortem have been substantiated on the subjective judgment of the dentist that could not be statistically quantified, through these dental patterns the evaluation is objective and easily interpretable. ${ }^{4,5}$

Martin-de-las-Heras et al. (2010) ${ }^{6}$ suggested a statistical method similar to the technique used for identification by mitochondrial DNA in which all of the comparisons between homologue pairs are performed against large databases of dental patterns. This approach supports the generation of general frequencies of 
combination and the consequent derivation of dental diversity patterns that enable high levels of identification. Biazevic et al. (2011) ${ }^{7}$ analyzed the national survey of oral health performed in Brazil in 2003, verified the dental diversity patterns in the Brazilian population, and analyzed the application of these patterns in different age groups (adolescents, adults, and elderly individuals) using the total and conditional diversity. They concluded that this epidemiological analysis improved the utility of dental patterns as an auxiliary scientific method for human identification. Based on analysis of large datasets, it is possible to verify dental diversity of a population. ${ }^{3,4,8}$ In Brazil, The National Oral Health Survey carried out in 2010 verified the oral health condition of Brazilian people and found that the DMF (decayed, missing, and filled teeth) index of Brazilian adolescents had decreased from 6.1 in the survey performed in 2003 to 4.2 , a 30\% reduction. ${ }^{9,10}$ This finding motivated the present study, as it was necessary to determine whether this increase in the number of higid teeth could reduce the dental diversity among adolescents and thus compromise the discrimination potential of dental patterns in human identification. This research aimed to ascertain whether the improvement in the oral health and thus the dental profile of Brazilian adolescents (aged 15-19 years) would interfere with the use of established dental diversity patterns and its potential use for human identification with the larger goal of using cross - sectional studies of oral health to obtain parameters that are both accurate and suitable for forensic use.

\section{MATERIALS AND METHODS}

Brazil has an extensive territory, with $8,511,767,049 \mathrm{~km}^{2}$, divided into different five geographic regions, in which ethnic diversity and socio-economic inequality are strongly pronounced. The diversity of dental patterns of Brazilian adolescents (15 to 19 years) was analyzed using secondary data from the last two National Oral Health Surveys that recorded the following dental conditions and other oral health conditions: decayed tooth, periodontal condition, dental trauma, occlusion, dental fluorosis, use and necessity of prosthesis, socio-economic condition, use of dental care services, and oral health selfperception. The original sample was divided into 5 age groups $(5,12,15$ to 19,35 to 44 and 65 to 74 years). These parameters of age groups, dental and oral health conditions were chosen in accordance with the criteria established by the World Health Organization (WHO). ${ }^{9,10}$

The representative sample of 15-to-19-year-old individuals chosen as per WHO parameters - included in the epidemiologic survey comprised 16,833 subjects in 2003 and 5,367 in 2010. The data related to each of the 28 dental units, 14 teeth in the upper arch (17-27) and 14 in the lower arch (37-47), were described, and the condition of each tooth was verified and recorded as one of following: higid (H), decayed (D), filled (F), missing $(\mathrm{M})$ or prosthesis $(\mathrm{P})$. Intact teeth without caries were classified as $\mathrm{H}$, teeth with some caries lesion(s) evident as $\mathrm{D}$, filled teeth as F, teeth missing due to caries or another reason as $\mathrm{M}$, and any total, removable, fixed prosthesis or dental crown as P. Non - erupted teeth, teeth with sealants, teeth with trauma, deciduous teeth, and teeth that could not be examined during the epidemiologic surveys for any reason were excluded. ${ }^{9,10}$

The coincidences observed in pairs of homologous teeth were classified according to the clinical condition ( $\mathrm{H}, \mathrm{D}, \mathrm{M}$, F, or P) and the subject's gender. The ordinary findings (present in more than $10 \%$ of the pairs) and extraordinary findings (present in less than $10 \%$ of the pairs) were described. Finally, the total diversity and the conditional diversity (calculated after the exclusion of all edentulous individuals and individuals with no missing or filled tooth) were estimated.

The original database was made available by the Health Ministry and included all of the information for all of the index ages and age groups studied in the national epidemiologic surveys. First, a new database was created that included only the information pertinent to the age group of interest (15-19 years of age). These data were transferred to a new spreadsheet using Microsoft Excel. The elaborated spreadsheet was then exported to the STATA 12.0 Program, which was used to prepare the database for the analysis. The national surveys of 2003 and 2010 had been submitted to and approved by the National Committee of Ethics in Research (CONEP).

\section{RESULTS}

The surveys of 2003 and 2010 included, respectively, 7,015 (41.67\%) and 2,497 (45.85\%) male and 9,818 (58.33\%) and 2,948 (54.15\%) female adolescents. Tables 1 and 2 compared to the results related to dental condition obtained from the 2003 Brazilian national oral health survey ${ }^{7}$ show that the frequency of condition $\mathrm{H}$ increased between 2003 and 2010 for all of the teeth analyzed. The largest changes were for teeth 37 and 47, for which the frequency of $\mathrm{H}$ increased, respectively, from $51.06 \%$ to $84.39 \%$ and from $52.21 \%$ to $85.41 \%$ in males and from $46.11 \%$ to $82.35 \%$ and from $47.28 \%$ to $84.19 \%$ in females. However, the first molars continued to be the teeth least likely to present with condition $\mathrm{H}$ for both genders.

The frequencies of the other conditions decreased for all teeth in both genders, with a few exceptions. In males, the frequency of condition $\mathrm{M}$ increased for teeth 31 and 44 and the frequency of $\mathrm{P}$ for teeth 17, 27, and 33, while the frequency of $\mathrm{P}$ did not change for teeth 25,31 , and 44 . In females, the frequency of condition $\mathrm{M}$ increased for teeth 34 and 44, while the frequency of P increased for tooth 21 and did not change for tooth 23. 
In addition, the largest changes in the frequency of condition $\mathrm{M}$ were for the first lower molars and the largest changes in the frequency of condition $\mathrm{F}$ for the second molars in both genders.

The greatest coincidence among the homologous pairs was found for condition $\mathrm{H}$ (Table 3) in both genders. However, the analysis revealed that unlike the situation in $2003^{7}$, in which there were a few coincidences of $\mathrm{P}$, in 2010 there were no coincidences of this condition among the males surveyed and only 2 cases of coincidence of homologous pairs among the females. The coincidence of condition $\mathrm{M}$ increased for pairs 1424 and 34-44 and decreased for the remaining pairs in both genders, between 2003 and 2010.

In the male adolescents (Table 4), comparing with results of the 2003 survey $^{7}$, we observed differences in the ordinary and extraordinary findings for teeth 11, 17, 21, 27, 31, 37, 41, 46, and 47 . The ordinary findings included changes from $\mathrm{HD}$ to $\mathrm{H}$ (teeth 11, 21, 31, and 41), from HDF to $\mathrm{H}$ (teeth 17, 27, and 47), from HDFM to HDF (tooth 46), and from HDF to $\mathrm{H}$ (tooth 27); the extraordinary findings included changes from MP to DMFP (teeth 11, 17, 27, and 47), from MFP to DMFP (teeth 21, 31, and 41), from P to MP (tooth 46), and from P to DMFP (tooth 37).

Among the female adolescents (Table 4), comparing with results of the 2003 survey $^{7}$, we observed differences in the ordinary and extraordinary findings for teeth $17,27,37,46$, and 47 . The conditions observed with greatest frequency (greater than 10\%) included changes from HDF to $\mathrm{H}$ (teeth 17, 27, and 47), from HDFM to HDF (tooth 46), and from HDF to HD (tooth 37), while the conditions observed with lowest frequency included changes from MP to DMFP (teeth 17, 27, and 47), from P to MP (tooth 46), and from MP to MFP (tooth 37).

Data from the last National Oral Health Survey point that the adolescents exhibited a low prevalence of edentulism (0.04\%) and a high rate of higid teeth $(22.77 \%$ ) (Data not shown in the tables). The female subjects presented with this condition at a frequency of $11.79 \%$ and the males at a frequency of $10.98 \%$. This situation interfered with the analysis of the conditional diversity estimate (Table 5).

The dental diversity patterns are shown in Table 5. Among females, the total diversity (28 teeth) changed from 0.930 $(2003)^{7}$ to 0.928 (2010) and the conditional diversity (28 teeth) from $0.981(2003)^{7}$ to 0.971 (2010). Among males, the total diversity (28 teeth) changed from $0.897(2003)^{7}$ to 0.931 (2010) and the conditional diversity (28 teeth) from 0.997 $(2003)^{7}$ to $0.980(2010)$.

The conditional diversity patterns varied by region and ranged in females from 0.932 to 0.971 , with the highest values in the Midwest region and the lowest in the Northeast Region; in males, the highest conditional diversity (0.991) was found in the Southeast Region and the lowest (0.976) in the Northeast Region.

\section{DISCUSSION}

The use of epidemiology as a planning and evaluation tool through the performance of nationwide research surveys enabling the collection of primary data on oral disease represents an advance in health care services. ${ }^{11}$ The sampling plan for Brazilian adolescents decreased between the surveys of 2003 and 2010. While the first survey comprised 16,833 individuals (9,818 females and 7,015 males), the second included only 5,445 (2,948 females and 2,497 males) (data not shown in the tables). These differences are explained, among others, because the sample size in 2003 was calculated from caries estimates derived from the survey of 1986, as there was no sample models for other conditions, whereas in 2010 were used estimates for the average variance of the DMF index based on national oral health survey 2003 data. ${ }^{11}$

Females predominated in both surveys. 9,10 These differences are attributable to the fact that the sample sizes of the two epidemiologic surveys were calculated from different estimates. Despite the different sizes of the samples of adolescents in 2003 and 2010, the samples were appropriately representative and the necessary statistical power was achieved, assuring the attainment of important results. ${ }^{11}$

The teeth are recognized as one of the most individual characteristics of the human body. However, young people now experience fewer caries and require fewer restorative treatments than did previous generations. ${ }^{1}$ Our study confirmed this fact: the frequency of condition $\mathrm{H}$ had increased in 2010 and was the most frequently reported of the conditions included in the survey. The increase in the frequency of condition $\mathrm{H}$ and the concomitant decreases in the frequencies of the other DMFP conditions (with some exceptions) for all of the teeth analyzed in Brazilian adolescents of both genders confirm the declining incidence of caries and the decrease in the need for dental prostheses, trends favored by both increases in access to services that benefit this age group and socio-economic factors. ${ }^{11}$ The latter are important because social changes that minimize the inequalities in oral health and promote action related to the social determinants and common risk factors for dental disease tend to improve disease prevention. ${ }^{12}$

The fact that the greatest coincidence among the homologous pairs corresponded to condition $\mathrm{H}$ of the anterior teeth has the potential to complicate the use of these teeth for human identification, as the majority of the dental patterns in use are derived from treatments for caries. ${ }^{13}$ 
Typical antemortem dental records may include radiographs, dental charts, intraoral photographs, dental casts and notes. Dental radiographs are one of the most desirable pieces of antemortem evidence, but they are not always available, and the comparison of antemortem and postmortem data characteristics must be based on handwritten charts and notes. ${ }^{4}$ This investigation is specifically concerned with non-radiographic dental comparison.

The rarity or lack thereof of the dental condition presented is related to the ordinary and extraordinary findings in an individual, which allow empirical comparisons to a database and make dental diversity patterns an objective method of identification. ${ }^{4}$ The oral health of Brazilian adolescents has evolved, and this was reflected by the changes from 2003 to 2010 in the most $(\mathrm{H})$ and least $(\mathrm{D}, \mathrm{F}, \mathrm{M}$, and $\mathrm{P})$ common findings. The value of the total dental diversity patterns decreased between 2003 and 2010 for the female adolescents but increased for the males. The result for the female sex was expected, as the number of higid teeth present increased; the apparent paradox for the male sex can be explained by the smaller sample size and underrepresentation of male respondents (46\%) in the 2010 survey.

Due to the increased number of higid teeth, the conditional diversity was estimated by excluding the populations with conditions that complicate the diversity of the dental patterns (individuals with all higid teeth and edentulous individuals), which increased the discriminatory power to a level comparable with that of the sequence diversity of mitochondrial DNA. It must be considered, on the other hand, that dental conditions change over time, differently of DNA.

The conditional diversity values were greater than the total diversity values not only for the female and male populations in 2003 and 2010 but also for the regional populations in 2010 . Such dental diversity values have potential for use in human identification, as noted by Martin-de-las-Heras et al. (2010) ${ }^{6}$ in their analysis of some data from the three last oral health surveys of Spain (1993, 2000, and 2005).

The social inequities present in Brazil are evident from indicators of disease processes and the levels of access to services of the most vulnerable groups in several regions of the country. ${ }^{15}$ The analysis of the regional patterns of diversity (Table 5) found the lowest values of total diversity in the southern and southeastern regions, as the lowest caries experience rates among adolescents were concentrated in these regions in both 2010 and 2003, while the highest frequencies of caries were found in the northeastern and central-western regions. ${ }^{9,10}$

There is no consensus about the threshold value at which dental diversity patterns are considered adequate for forensic purposes, although the quantitative statistical model for DNA, a highly effective method, provides some basis for comparison. The diversity of mitochondrial DNA depends on the population studied; some regional estimates of diversity are 0.964 (Portugal), 0.930 (Costa Rica), 0.981 (Mexico), 0.989 (USA), 0.997 (North American populations), and 0.997 (Spain). ${ }^{6}$ Our research found that the patterns were more diverse for some of the populations examined, such as those in which all of the teeth were present, and less diverse for others, such as when only the superior or the inferior teeth were available, a not-uncommon situation after a mass disaster event. Other dental methods can also be used in these cases. ${ }^{16}$

One problem that limits the use of dental pattern diversity for identifying human remains is the insufficient quantity or quality of ante-mortem dental data. ${ }^{1,7,17}$ Other limitations of the present study include the lack of regional tables for the comparisons and the lack of data for other age groups that were not included in the epidemiologic surveys. Also, the protocol for human identification states that ante - and post - mortem data be compared; it's necessary to update dental information over time, since dental characteristics change due to natural progression. Identification by means of dental diversity will also require standardization of dental data records so that they can be analyzed and interpreted by forensic dentists in several countries. ${ }^{18}$ This would increase the efficiency of the investigations and thus the potential for identifying victims after mass disasters. In addition, a national record of dental characteristics could help with the identification of missing people, as reported in Australia and in other countries. ${ }^{19}$ The decrease in the conditional diversity patterns that occurred in both genders between 2003 and 2010 resulted from improvement in the oral health condition of Brazilian adolescents. ${ }^{9,10}$ Although this does not yet interfere with the potential of this method, the data indicate a progressive decrease in the frequency of teeth with dental treatments and a consequent decrease in the discriminating power of diversity patterns for human identification. These trends will necessitate both the development of other methodologies to potentiate the use of forensic dentistry and the selection of other dental morphological characteristics besides restorations for inclusion in future epidemiologic surveys in order to maintain the usefulness of dental characteristics for human identification.

At the end of this study, it is notable that the improvement of oral health condition in adolescents didn't interfere with the discriminant power of dental diversity patterns.

\section{ACKNOWLEDGEMENT}

This work was made possible thanks to funding from CAPES (Brazilian Coordination for the Improvement of Higher Education Personnel). 
Table 1. The distribution of teeth according to dental condition and age group in males $(H=$ higid; $D=$ decayed; $M=$ missed; $F=$ filled; $P=$ prosthesis $)$

\begin{tabular}{|c|c|c|c|c|c|c|c|c|c|c|c|c|c|c|c|c|}
\hline \multicolumn{17}{|c|}{ Adolescent (Male)* - 2003} \\
\hline $\mathbf{H}$ & $\begin{array}{c}1211 \\
(86.07)\end{array}$ & $\begin{array}{c}4639 \\
(67.81) \\
\end{array}$ & $\begin{array}{c}3266 \\
(47.42)\end{array}$ & $\begin{array}{c}5765 \\
(84.28) \\
\end{array}$ & $\begin{array}{c}5769 \\
(84.42) \\
\end{array}$ & $\begin{array}{c}6739 \\
(96.59)\end{array}$ & $\begin{array}{c}5942 \\
(85.31) \\
\end{array}$ & $\begin{array}{c}5624 \\
(80.63) \\
\end{array}$ & $\begin{array}{c}5600 \\
(80.22) \\
\end{array}$ & $\begin{array}{c}5896 \\
(84.64) \\
\end{array}$ & $\begin{array}{c}6735 \\
(96.49) \\
\end{array}$ & $\begin{array}{c}5801 \\
(84.79) \\
\end{array}$ & $\begin{array}{l}5756 \\
(84.23) \\
\end{array}$ & $\begin{array}{c}3241 \\
(47.11) \\
\end{array}$ & $\begin{array}{c}4584 \\
(66.77)\end{array}$ & $\begin{array}{c}1189 \\
(86.1)\end{array}$ \\
\hline D & $\begin{array}{c}102 \\
(7.25)\end{array}$ & $\begin{array}{l}1275 \\
(8.64)\end{array}$ & $\begin{array}{c}1390 \\
(20.18)\end{array}$ & $\begin{array}{c}463 \\
(6.77)\end{array}$ & $\begin{array}{c}434 \\
(6.35)\end{array}$ & $\begin{array}{c}127 \\
(1.82)\end{array}$ & $\begin{array}{c}574 \\
(8.24)\end{array}$ & $\begin{array}{c}720 \\
(10.32)\end{array}$ & $\begin{array}{c}730 \\
(10.46)\end{array}$ & $\begin{array}{c}589 \\
(8.46)\end{array}$ & $\begin{array}{c}136 \\
(1.95)\end{array}$ & $\begin{array}{c}411 \\
(6.01)\end{array}$ & $\begin{array}{c}454 \\
(6.64)\end{array}$ & $\begin{array}{c}1479 \\
(21.5)\end{array}$ & $\begin{array}{c}1306 \\
(19.02)\end{array}$ & $\begin{array}{c}81 \\
(5.87)\end{array}$ \\
\hline $\mathbf{F}$ & $\begin{array}{c}36 \\
(2.56)\end{array}$ & $\begin{array}{c}801 \\
(11.71) \\
\end{array}$ & $\begin{array}{c}1758 \\
(25.52)\end{array}$ & $\begin{array}{c}502 \\
(7.34)\end{array}$ & $\begin{array}{c}475 \\
(6.95) \\
\end{array}$ & $\begin{array}{c}69 \\
(0.99) \\
\end{array}$ & $\begin{array}{c}347 \\
(4.98)\end{array}$ & $\begin{array}{c}498 \\
(7.14)\end{array}$ & $\begin{array}{c}513 \\
(7.35)\end{array}$ & $\begin{array}{c}367 \\
(5.27)\end{array}$ & $\begin{array}{l}70 \\
(1)\end{array}$ & $\begin{array}{c}481 \\
(7.03)\end{array}$ & $\begin{array}{c}506 \\
(7.4)\end{array}$ & $\begin{array}{c}1728 \\
(25.12) \\
\end{array}$ & $\begin{array}{c}851 \\
(12.4)\end{array}$ & $\begin{array}{c}42 \\
(3.04)\end{array}$ \\
\hline $\mathbf{M}$ & $\begin{array}{c}56 \\
(3.98) \\
\end{array}$ & $\begin{array}{c}121 \\
(1.77) \\
\end{array}$ & $\begin{array}{c}467 \\
(6.78) \\
\end{array}$ & $\begin{array}{c}105 \\
(1.54) \\
\end{array}$ & $\begin{array}{c}150 \\
(2.19) \\
\end{array}$ & $\begin{array}{c}38 \\
(0.54) \\
\end{array}$ & $\begin{array}{c}95 \\
(1.36) \\
\end{array}$ & $\begin{array}{c}116 \\
(1.66) \\
\end{array}$ & $\begin{array}{c}123 \\
(1.76) \\
\end{array}$ & $\begin{array}{c}104 \\
(1.49) \\
\end{array}$ & $\begin{array}{c}36 \\
(0.52) \\
\end{array}$ & $\begin{array}{c}143 \\
(2.09) \\
\end{array}$ & $\begin{array}{c}114 \\
(1.67) \\
\end{array}$ & $\begin{array}{c}426 \\
(6.19) \\
\end{array}$ & $\begin{array}{c}121 \\
(1.76) \\
\end{array}$ & $\begin{array}{c}66 \\
(4.78) \\
\end{array}$ \\
\hline $\mathbf{P}$ & $\begin{array}{c}2 \\
(0.14)\end{array}$ & $\begin{array}{c}5 \\
(0.07) \\
\end{array}$ & $\begin{array}{c}7 \\
(0.1)\end{array}$ & $\begin{array}{c}5 \\
(0.07) \\
\end{array}$ & $\begin{array}{c}6 \\
(0.09)\end{array}$ & $\begin{array}{c}4 \\
(0.06) \\
\end{array}$ & $\begin{array}{c}7 \\
(0.1) \\
\end{array}$ & $\begin{array}{c}17 \\
(0.24) \\
\end{array}$ & $\begin{array}{c}15 \\
(0.21) \\
\end{array}$ & $\begin{array}{c}10 \\
(0.14)\end{array}$ & $\begin{array}{c}3 \\
(0.04) \\
\end{array}$ & $\begin{array}{c}6 \\
(0.09) \\
\end{array}$ & $\begin{array}{c}4 \\
(0.06) \\
\end{array}$ & $\begin{array}{c}6 \\
(0.09)\end{array}$ & $\begin{array}{c}3 \\
(0.04) \\
\end{array}$ & $\begin{array}{c}3 \\
(0.22) \\
\end{array}$ \\
\hline & 18 & 17 & 16 & 15 & 14 & 13 & 12 & 11 & 21 & 22 & 23 & 24 & 25 & 26 & 27 & 28 \\
\hline & 48 & 47 & 46 & 45 & 44 & 43 & 42 & 41 & 31 & 32 & 33 & 34 & 35 & 36 & 37 & 38 \\
\hline $\mathbf{H}$ & $\begin{array}{c}1547 \\
(84.21)\end{array}$ & $\begin{array}{c}3579 \\
(52.21) \\
\end{array}$ & $\begin{array}{c}2261 \\
(32.87)\end{array}$ & $\begin{array}{c}5871 \\
(85.71) \\
\end{array}$ & $\begin{array}{c}6375 \\
(92.79) \\
\end{array}$ & $\begin{array}{c}6924 \\
(98.82) \\
\end{array}$ & $\begin{array}{c}6887 \\
(98.33) \\
\end{array}$ & $\begin{array}{c}6858 \\
(98.04) \\
\end{array}$ & $\begin{array}{c}6870 \\
(98.06) \\
\end{array}$ & $\begin{array}{c}6885 \\
(98.26) \\
\end{array}$ & $\begin{array}{c}6940 \\
(99.04) \\
\end{array}$ & $\begin{array}{c}6391 \\
(92.96) \\
\end{array}$ & $\begin{array}{c}5832 \\
(85.06) \\
\end{array}$ & $\begin{array}{c}2264 \\
(32.9) \\
\end{array}$ & $\begin{array}{c}3508 \\
(51.06) \\
\end{array}$ & $\begin{array}{c}1541 \\
(85.04) \\
\end{array}$ \\
\hline D & $\begin{array}{c}138 \\
(7.51)\end{array}$ & $\begin{array}{c}1768 \\
(25.79)\end{array}$ & $\begin{array}{c}1525 \\
(22.17)\end{array}$ & $\begin{array}{c}393 \\
(5.74)\end{array}$ & $\begin{array}{c}162 \\
(2.36)\end{array}$ & $\begin{array}{c}48 \\
(0.69) \\
\end{array}$ & $\begin{array}{c}76 \\
(1.09) \\
\end{array}$ & $\begin{array}{c}89 \\
(1.27) \\
\end{array}$ & $\begin{array}{c}89 \\
(1.27) \\
\end{array}$ & $\begin{array}{c}75 \\
(1.07)\end{array}$ & $\begin{array}{c}34 \\
(0.49) \\
\end{array}$ & $\begin{array}{c}169 \\
(2.46)\end{array}$ & $\begin{array}{c}425 \\
(6.2) \\
\end{array}$ & $\begin{array}{c}1493 \\
(21.69)\end{array}$ & $\begin{array}{c}1860 \\
(27.07)\end{array}$ & $\begin{array}{c}130 \\
(7.17)\end{array}$ \\
\hline $\mathbf{F}$ & $\begin{array}{c}70 \\
(3.81)\end{array}$ & $\begin{array}{c}1225 \\
(17.87)\end{array}$ & $\begin{array}{c}1878 \\
(27.3) \\
\end{array}$ & $\begin{array}{c}454 \\
(6.63)\end{array}$ & $\begin{array}{c}261 \\
(3.8)\end{array}$ & $\begin{array}{c}21 \\
(0.3)\end{array}$ & $\begin{array}{c}22 \\
(0.31)\end{array}$ & $\begin{array}{c}36 \\
(0.51) \\
\end{array}$ & $\begin{array}{c}36 \\
(0.51)\end{array}$ & $\begin{array}{c}33 \\
(0.47) \\
\end{array}$ & $\begin{array}{c}22 \\
(0.31)\end{array}$ & $\begin{array}{c}237 \\
(3.45)\end{array}$ & $\begin{array}{c}474 \\
(6.91)\end{array}$ & $\begin{array}{c}1888 \\
(27.43) \\
\end{array}$ & $\begin{array}{c}1225 \\
(17.83)\end{array}$ & $\begin{array}{c}67 \\
(3.7)\end{array}$ \\
\hline $\mathbf{M}$ & $\begin{array}{c}79 \\
(4.3) \\
\end{array}$ & $\begin{array}{c}280 \\
(4.08) \\
\end{array}$ & $\begin{array}{c}1207 \\
(17.55)\end{array}$ & $\begin{array}{c}128 \\
(1.87) \\
\end{array}$ & $\begin{array}{l}69 \\
(1) \\
\end{array}$ & $\begin{array}{c}11 \\
(0.16) \\
\end{array}$ & $\begin{array}{c}16 \\
(0.23) \\
\end{array}$ & $\begin{array}{c}9 \\
(0.13) \\
\end{array}$ & $\begin{array}{c}8 \\
(0.11) \\
\end{array}$ & $\begin{array}{c}11 \\
(0.16)\end{array}$ & $\begin{array}{c}8 \\
(0.11) \\
\end{array}$ & $\begin{array}{c}75 \\
(1.09) \\
\end{array}$ & $\begin{array}{c}121 \\
(1.76)\end{array}$ & $\begin{array}{c}1234 \\
(17.93) \\
\end{array}$ & $\begin{array}{c}274 \\
(3.99) \\
\end{array}$ & $\begin{array}{c}71 \\
(3.92) \\
\end{array}$ \\
\hline $\mathbf{P}$ & $\begin{array}{c}3 \\
(0.16) \\
\end{array}$ & $\begin{array}{c}3 \\
(0.04) \\
\end{array}$ & $\begin{array}{c}8 \\
(0.12) \\
\end{array}$ & $\begin{array}{c}4 \\
(0.06) \\
\end{array}$ & $\begin{array}{c}3 \\
(0.04) \\
\end{array}$ & $\begin{array}{c}3 \\
(0.04) \\
\end{array}$ & $\begin{array}{c}3 \\
(0.04) \\
\end{array}$ & $\begin{array}{c}3 \\
(0.04) \\
\end{array}$ & $\begin{array}{c}3 \\
(0.04) \\
\end{array}$ & $\begin{array}{c}3 \\
(0.04) \\
\end{array}$ & $\begin{array}{c}3 \\
(0.04) \\
\end{array}$ & $\begin{array}{c}3 \\
(0.04) \\
\end{array}$ & $\begin{array}{c}4 \\
(0.06) \\
\end{array}$ & $\begin{array}{c}3 \\
(0.04) \\
\end{array}$ & $\begin{array}{c}3 \\
(0.04) \\
\end{array}$ & $\begin{array}{c}3 \\
(0.17) \\
\end{array}$ \\
\hline \multicolumn{17}{|c|}{ Adolescent (Male)* - 2010} \\
\hline $\mathbf{H}$ & $\begin{array}{c}180 \\
(97.83) \\
\end{array}$ & $\begin{array}{c}1097 \\
(92.97) \\
\end{array}$ & $\begin{array}{c}1197 \\
(69.23) \\
\end{array}$ & $\begin{array}{c}1764 \\
(92.7) \\
\end{array}$ & $\begin{array}{c}1715 \\
(90.5) \\
\end{array}$ & $\begin{array}{c}1947 \\
(98.78)\end{array}$ & $\begin{array}{c}1978 \\
(96.63) \\
\end{array}$ & $\begin{array}{c}2067 \\
(87.36) \\
\end{array}$ & $\begin{array}{c}2103 \\
(88.81) \\
\end{array}$ & $\begin{array}{c}2018 \\
(96.6) \\
\end{array}$ & $\begin{array}{c}1996 \\
(99.11) \\
\end{array}$ & $\begin{array}{c}1771 \\
(91.05) \\
\end{array}$ & $\begin{array}{c}1671 \\
(94.78) \\
\end{array}$ & $\begin{array}{c}1149 \\
(70.02) \\
\end{array}$ & $\begin{array}{c}1057 \\
(93.21) \\
\end{array}$ & $\begin{array}{c}207 \\
(88.46) \\
\end{array}$ \\
\hline D & $\begin{array}{c}2 \\
(1.09) \\
\end{array}$ & $\begin{array}{c}58 \\
(4.92) \\
\end{array}$ & $\begin{array}{c}202 \\
(11.68) \\
\end{array}$ & $\begin{array}{c}70 \\
(3.68) \\
\end{array}$ & $\begin{array}{c}64 \\
(3.38) \\
\end{array}$ & $\begin{array}{c}15 \\
(0.76) \\
\end{array}$ & $\begin{array}{c}37 \\
(1.81) \\
\end{array}$ & $\begin{array}{c}163 \\
(6.89) \\
\end{array}$ & $\begin{array}{c}123 \\
(5.19) \\
\end{array}$ & $\begin{array}{c}43 \\
(2.06) \\
\end{array}$ & $\begin{array}{c}6 \\
(0.3) \\
\end{array}$ & $\begin{array}{c}58 \\
(2.98) \\
\end{array}$ & $\begin{array}{c}44 \\
(2.5) \\
\end{array}$ & $\begin{array}{c}182 \\
(11.09) \\
\end{array}$ & $\begin{array}{c}49 \\
(4.32) \\
\end{array}$ & $\begin{array}{c}3 \\
(1.28) \\
\end{array}$ \\
\hline $\mathbf{F}$ & $\begin{array}{c}2 \\
(1.09) \\
\end{array}$ & $\begin{array}{c}23 \\
(1.95) \\
\end{array}$ & $\begin{array}{c}292 \\
(16.89) \\
\end{array}$ & $\begin{array}{c}50 \\
(2.63) \\
\end{array}$ & $\begin{array}{c}77 \\
(4.06) \\
\end{array}$ & $\begin{array}{c}5 \\
(0.25) \\
\end{array}$ & $\begin{array}{c}25 \\
(1.22) \\
\end{array}$ & $\begin{array}{c}120 \\
(5.07) \\
\end{array}$ & $\begin{array}{c}128 \\
(5.41) \\
\end{array}$ & $\begin{array}{c}23 \\
(1.1) \\
\end{array}$ & $\begin{array}{c}5 \\
(0.25) \\
\end{array}$ & $\begin{array}{c}85 \\
(4.37) \\
\end{array}$ & $\begin{array}{c}33 \\
(1.87) \\
\end{array}$ & $\begin{array}{c}270 \\
(16.45) \\
\end{array}$ & $\begin{array}{c}24 \\
(2.12) \\
\end{array}$ & $\begin{array}{c}3 \\
(1.28) \\
\end{array}$ \\
\hline $\mathbf{M}$ & $\begin{array}{c}0 \\
(0) \\
\end{array}$ & $\begin{array}{c}1 \\
(0.08) \\
\end{array}$ & $\begin{array}{c}38 \\
(2.2) \\
\end{array}$ & $\begin{array}{c}18 \\
(0.95) \\
\end{array}$ & $\begin{array}{c}39 \\
(2.06) \\
\end{array}$ & $\begin{array}{c}4 \\
(0.2) \\
\end{array}$ & $\begin{array}{c}7 \\
(0.34) \\
\end{array}$ & $\begin{array}{c}15 \\
(0.63) \\
\end{array}$ & $\begin{array}{c}13 \\
(0.55) \\
\end{array}$ & $\begin{array}{c}5 \\
(0.24) \\
\end{array}$ & $\begin{array}{c}7 \\
(0.35) \\
\end{array}$ & $\begin{array}{c}31 \\
(1.59) \\
\end{array}$ & $\begin{array}{c}14 \\
(0.79) \\
\end{array}$ & $\begin{array}{c}40 \\
(2.44) \\
\end{array}$ & $\begin{array}{c}3 \\
(0.26) \\
\end{array}$ & $\begin{array}{c}21 \\
(8.97) \\
\end{array}$ \\
\hline \multirow[t]{3}{*}{$\mathbf{P}$} & $\begin{array}{c}0 \\
0 \\
(0)\end{array}$ & $\begin{array}{c}1 \\
(0.08) \\
\end{array}$ & $\begin{array}{c}0 \\
(0) \\
\end{array}$ & $\begin{array}{c}1 \\
(0.05) \\
\end{array}$ & $\begin{array}{c}0 \\
(0) \\
\end{array}$ & $\begin{array}{c}0 \\
(0) \\
\end{array}$ & $\begin{array}{c}0 \\
(0) \\
\end{array}$ & $\begin{array}{c}1 \\
(0.04) \\
\end{array}$ & $\begin{array}{c}1 \\
(0.04) \\
\end{array}$ & $\begin{array}{c}0 \\
(0) \\
\end{array}$ & $\begin{array}{c}0 \\
(0) \\
\end{array}$ & $\begin{array}{c}0 \\
(0) \\
\end{array}$ & $\begin{array}{c}1 \\
(0.06) \\
\end{array}$ & $\begin{array}{c}0 \\
(0) \\
\end{array}$ & $\begin{array}{c}1 \\
(0.09) \\
\end{array}$ & $\begin{array}{c}0 \\
(0) \\
\end{array}$ \\
\hline & 18 & 17 & 16 & 15 & 14 & 13 & 12 & 11 & 21 & 22 & 23 & 24 & 25 & 26 & 27 & 28 \\
\hline & 48 & 47 & 46 & 45 & 44 & 43 & 42 & 41 & 31 & 32 & 33 & 34 & 35 & 36 & 37 & 38 \\
\hline $\mathbf{H}$ & $\begin{array}{c}231 \\
(91.3)\end{array}$ & $\begin{array}{c}890 \\
(85.41)\end{array}$ & $\begin{array}{c}1052 \\
(52.52) \\
\end{array}$ & $\begin{array}{c}2042 \\
(94.41) \\
\end{array}$ & $\begin{array}{c}2183 \\
(94.46) \\
\end{array}$ & $\begin{array}{c}2353 \\
(99.32) \\
\end{array}$ & $\begin{array}{c}2373 \\
(99.29) \\
\end{array}$ & $\begin{array}{l}2395 \\
(98.4) \\
\end{array}$ & $\begin{array}{c}2412 \\
(98.89) \\
\end{array}$ & $\begin{array}{c}2394 \\
(99.42) \\
\end{array}$ & $\begin{array}{c}2378 \\
(99.29) \\
\end{array}$ & $\begin{array}{c}2217 \\
(94.99) \\
\end{array}$ & $\begin{array}{c}2056 \\
(93.37) \\
\end{array}$ & $\begin{array}{c}1099 \\
(54.68) \\
\end{array}$ & $\begin{array}{c}919 \\
(84.39)\end{array}$ & $\begin{array}{c}221 \\
(88.76)\end{array}$ \\
\hline D & $\begin{array}{c}1 \\
(0.4)\end{array}$ & $\begin{array}{c}96 \\
(9.21) \\
\end{array}$ & $\begin{array}{c}308 \\
(15.38) \\
\end{array}$ & $\begin{array}{c}49 \\
(2.27) \\
\end{array}$ & $\begin{array}{c}33 \\
(1.43)\end{array}$ & $\begin{array}{c}8 \\
(0.34) \\
\end{array}$ & $\begin{array}{c}7 \\
(0.29) \\
\end{array}$ & $\begin{array}{c}25 \\
(1.03)\end{array}$ & $\begin{array}{c}15 \\
(0.62)\end{array}$ & $\begin{array}{c}10 \\
(0.42)\end{array}$ & $\begin{array}{c}11 \\
(0.46)\end{array}$ & $\begin{array}{c}37 \\
(1.59)\end{array}$ & $\begin{array}{c}52 \\
(2.36) \\
\end{array}$ & $\begin{array}{c}306 \\
(15.22) \\
\end{array}$ & $\begin{array}{c}103 \\
(9.46)\end{array}$ & $\begin{array}{c}5 \\
(2.01)\end{array}$ \\
\hline $\mathbf{F}$ & $\begin{array}{c}2 \\
(0.79) \\
\end{array}$ & $\begin{array}{c}44 \\
(4.22) \\
\end{array}$ & $\begin{array}{c}513 \\
(25.61) \\
\end{array}$ & $\begin{array}{c}54 \\
(2.5) \\
\end{array}$ & $\begin{array}{c}62 \\
(2.68) \\
\end{array}$ & $\begin{array}{c}5 \\
(0.21) \\
\end{array}$ & $\begin{array}{c}7 \\
(0.29) \\
\end{array}$ & $\begin{array}{c}12 \\
(0.49) \\
\end{array}$ & $\begin{array}{c}7 \\
(0.29) \\
\end{array}$ & $\begin{array}{c}2 \\
(0.08) \\
\end{array}$ & $\begin{array}{c}3 \\
(0.13) \\
\end{array}$ & $\begin{array}{c}56 \\
(2.4) \\
\end{array}$ & $\begin{array}{c}69 \\
(3.13) \\
\end{array}$ & $\begin{array}{c}470 \\
(23.38) \\
\end{array}$ & $\begin{array}{c}59 \\
(5.42) \\
\end{array}$ & $\begin{array}{c}2 \\
(0.8)\end{array}$ \\
\hline $\mathbf{M}$ & $\begin{array}{c}19 \\
(7.51)\end{array}$ & $\begin{array}{c}12 \\
(1.15)\end{array}$ & $\begin{array}{c}130 \\
(6.49)\end{array}$ & $\begin{array}{c}18 \\
(0.83)\end{array}$ & $\begin{array}{c}32 \\
(1.38)\end{array}$ & $\begin{array}{c}3 \\
(0.13) \\
\end{array}$ & $\begin{array}{c}3 \\
(0.13)\end{array}$ & $\begin{array}{c}2 \\
(0.08)\end{array}$ & $\begin{array}{c}4 \\
(0.16)\end{array}$ & $\begin{array}{c}2 \\
(0.08)\end{array}$ & $\begin{array}{c}1 \\
(0.04)\end{array}$ & $\begin{array}{c}24 \\
(1.03)\end{array}$ & $\begin{array}{c}25 \\
(1.14)\end{array}$ & $\begin{array}{c}135 \\
(6.72)\end{array}$ & $\begin{array}{c}8 \\
(0.73)\end{array}$ & $\begin{array}{c}21 \\
(8.43)\end{array}$ \\
\hline $\mathbf{P}$ & $\begin{array}{c}0 \\
(0)\end{array}$ & $\begin{array}{c}0 \\
(0)\end{array}$ & $\begin{array}{c}0 \\
(0)\end{array}$ & $\begin{array}{c}0 \\
(0)\end{array}$ & $\begin{array}{c}1 \\
(0.04)\end{array}$ & $\begin{array}{c}0 \\
(0)\end{array}$ & $\begin{array}{c}0 \\
(0) \\
\end{array}$ & $\begin{array}{c}0 \\
(0)\end{array}$ & $\begin{array}{c}1 \\
(0.04)\end{array}$ & $\begin{array}{c}0 \\
(0)\end{array}$ & $\begin{array}{c}2 \\
(0.08)\end{array}$ & $\begin{array}{c}0 \\
(0) \\
\end{array}$ & $\begin{array}{c}0 \\
(0)\end{array}$ & $\begin{array}{c}0 \\
(0)\end{array}$ & $\begin{array}{c}0 \\
(0)\end{array}$ & $\begin{array}{c}0 \\
(0)\end{array}$ \\
\hline
\end{tabular}

${ }^{*} \mathrm{p}<0.001$ Association between sample and codes. 
Table 2. The distribution of teeth according to dental condition and age group in females $(H=$ higid; $D=$ decayed; $M=$ missed; $F=$ filled; $P=$ prosthesis)

\begin{tabular}{|c|c|c|c|c|c|c|c|c|c|c|c|c|c|c|c|c|}
\hline \multicolumn{17}{|c|}{ Adolescent $\left(\right.$ Female) ${ }^{*}-2003$} \\
\hline $\mathbf{H}$ & $\begin{array}{c}1635 \\
(82.53)\end{array}$ & $\begin{array}{c}6016 \\
(62.65)\end{array}$ & $\begin{array}{c}4396 \\
(45.63)\end{array}$ & $\begin{array}{c}7805 \\
(81.86)\end{array}$ & $\begin{array}{c}7838 \\
(82.19)\end{array}$ & $\begin{array}{c}9437 \\
(96.45)\end{array}$ & $\begin{array}{c}8269 \\
(84.76)\end{array}$ & $\begin{array}{c}7862 \\
(80.47)\end{array}$ & $\begin{array}{c}7822 \\
(79.98)\end{array}$ & $\begin{array}{c}8195 \\
(83.93)\end{array}$ & $\begin{array}{c}9390 \\
(96.06)\end{array}$ & $\begin{array}{c}7828 \\
(81.99)\end{array}$ & $\begin{array}{c}7748 \\
(81.24)\end{array}$ & $\begin{array}{c}4350 \\
(45.1)\end{array}$ & $\begin{array}{c}5974 \\
(62.15)\end{array}$ & $\begin{array}{c}1612 \\
(81.66)\end{array}$ \\
\hline D & $\begin{array}{c}158 \\
(7.98)\end{array}$ & $\begin{array}{c}1864 \\
(19.41)\end{array}$ & $\begin{array}{c}1778 \\
(18.45)\end{array}$ & $\begin{array}{c}630 \\
(6.61)\end{array}$ & $\begin{array}{c}605 \\
(6.34)\end{array}$ & $\begin{array}{c}166 \\
(1.7)\end{array}$ & $\begin{array}{c}764 \\
(7.83)\end{array}$ & $\begin{array}{c}899 \\
(9.2)\end{array}$ & $\begin{array}{c}933 \\
(9.54)\end{array}$ & $\begin{array}{c}801 \\
(8.2)\end{array}$ & $\begin{array}{c}188 \\
(1.92)\end{array}$ & $\begin{array}{c}657 \\
(6.88)\end{array}$ & $\begin{array}{c}705 \\
(7.39)\end{array}$ & $\begin{array}{c}1836 \\
(19.04)\end{array}$ & $\begin{array}{c}1917 \\
(19.94)\end{array}$ & $\begin{array}{c}165 \\
(8.36)\end{array}$ \\
\hline $\mathbf{F}$ & $\begin{array}{c}58 \\
(2.93) \\
\end{array}$ & $\begin{array}{c}1530 \\
(15.93)\end{array}$ & $\begin{array}{c}2691 \\
(27.93)\end{array}$ & $\begin{array}{c}881 \\
(9.24) \\
\end{array}$ & $\begin{array}{c}827 \\
(8.67) \\
\end{array}$ & $\begin{array}{c}102 \\
(1.04)\end{array}$ & $\begin{array}{c}522 \\
(5.35) \\
\end{array}$ & $\begin{array}{c}783 \\
(8.01) \\
\end{array}$ & $\begin{array}{c}799 \\
(8.17) \\
\end{array}$ & $\begin{array}{c}558 \\
(5.71) \\
\end{array}$ & $\begin{array}{c}115 \\
(1.18)\end{array}$ & $\begin{array}{c}820 \\
(8.59) \\
\end{array}$ & $\begin{array}{c}865 \\
(9.07) \\
\end{array}$ & $\begin{array}{c}2758 \\
(28.6)\end{array}$ & $\begin{array}{c}1521 \\
(15.82)\end{array}$ & $\begin{array}{c}67 \\
(3.39) \\
\end{array}$ \\
\hline M & $\begin{array}{c}126 \\
(6.36)\end{array}$ & $\begin{array}{c}188 \\
(1.96)\end{array}$ & $\begin{array}{c}763 \\
(7.92)\end{array}$ & $\begin{array}{c}214 \\
(2.24)\end{array}$ & $\begin{array}{c}259 \\
(2.72)\end{array}$ & $\begin{array}{c}74 \\
(0.76) \\
\end{array}$ & $\begin{array}{c}196 \\
(2.01)\end{array}$ & $\begin{array}{l}205 \\
(2.1)\end{array}$ & $\begin{array}{c}209 \\
(2.14)\end{array}$ & $\begin{array}{c}203 \\
(2.08)\end{array}$ & $\begin{array}{c}78 \\
(0.8)\end{array}$ & $\begin{array}{c}236 \\
(2.47)\end{array}$ & $\begin{array}{c}212 \\
(2.22)\end{array}$ & $\begin{array}{c}693 \\
(7.19)\end{array}$ & $\begin{array}{c}197 \\
(2.05)\end{array}$ & $\begin{array}{c}126 \\
(6.38)\end{array}$ \\
\hline $\mathbf{P}$ & $\begin{array}{c}4 \\
(0.2) \\
\end{array}$ & $\begin{array}{c}4 \\
(0.04)\end{array}$ & $\begin{array}{c}7 \\
(0.07) \\
\end{array}$ & $\begin{array}{c}4 \\
(0.04) \\
\end{array}$ & $\begin{array}{c}7 \\
(0.07) \\
\end{array}$ & $\begin{array}{c}5 \\
(0.05) \\
\end{array}$ & $\begin{array}{c}5 \\
(0.05) \\
\end{array}$ & $\begin{array}{c}21 \\
(0.21)\end{array}$ & $\begin{array}{c}17 \\
(0.17)\end{array}$ & $\begin{array}{c}7 \\
(0.07) \\
\end{array}$ & $\begin{array}{c}4 \\
(0.04) \\
\end{array}$ & $\begin{array}{c}6 \\
(0.06) \\
\end{array}$ & $\begin{array}{c}7 \\
(0.07) \\
\end{array}$ & $\begin{array}{c}8 \\
(0.08) \\
\end{array}$ & $\begin{array}{c}3 \\
(0.03) \\
\end{array}$ & $\begin{array}{c}4 \\
(0.2)\end{array}$ \\
\hline & 18 & 17 & 16 & 15 & 14 & 13 & 12 & 11 & 21 & 22 & 23 & 24 & 25 & 26 & 27 & 28 \\
\hline & 48 & 47 & 46 & 45 & 44 & 43 & 42 & 41 & 31 & 32 & 33 & 34 & 35 & 36 & 37 & 38 \\
\hline $\mathbf{H}$ & $\begin{array}{c}1987 \\
(79.29)\end{array}$ & $\begin{array}{c}4541 \\
(47.28)\end{array}$ & $\begin{array}{c}2728 \\
(28.26) \\
\end{array}$ & $\begin{array}{c}7924 \\
(82.93)\end{array}$ & $\begin{array}{c}8870 \\
(92.88)\end{array}$ & $\begin{array}{c}9707 \\
(99.01)\end{array}$ & $\begin{array}{l}9682 \\
(98.8) \\
\end{array}$ & $\begin{array}{c}9659 \\
(98.59)\end{array}$ & $\begin{array}{c}9640 \\
(98.37)\end{array}$ & $\begin{array}{c}9677 \\
(98.69)\end{array}$ & $\begin{array}{c}9701 \\
(98.98)\end{array}$ & $\begin{array}{c}8847 \\
(92.48)\end{array}$ & $\begin{array}{c}7910 \\
(82.78)\end{array}$ & $\begin{array}{c}2658 \\
(27.56)\end{array}$ & $\begin{array}{c}4433 \\
(46.11)\end{array}$ & $\begin{array}{c}1963 \\
(79.35) \\
\end{array}$ \\
\hline D & $\begin{array}{c}250 \\
(9.98)\end{array}$ & $\begin{array}{c}2302 \\
(23.97) \\
\end{array}$ & $\begin{array}{c}1933 \\
(20.02)\end{array}$ & $\begin{array}{r}535 \\
(5.6) \\
\end{array}$ & $\begin{array}{c}197 \\
(2.06) \\
\end{array}$ & $\begin{array}{c}55 \\
(0.56) \\
\end{array}$ & $\begin{array}{c}70 \\
(0.71) \\
\end{array}$ & $\begin{array}{c}84 \\
(0.86) \\
\end{array}$ & $\begin{array}{c}99 \\
(1.01) \\
\end{array}$ & $\begin{array}{c}89 \\
(0.91) \\
\end{array}$ & $\begin{array}{c}63 \\
(0.64) \\
\end{array}$ & $\begin{array}{c}231 \\
(2.41) \\
\end{array}$ & $\begin{array}{c}552 \\
(5.78) \\
\end{array}$ & $\begin{array}{c}1952 \\
(20.24) \\
\end{array}$ & $\begin{array}{c}2359 \\
(24.53) \\
\end{array}$ & $\begin{array}{c}258 \\
(10.43) \\
\end{array}$ \\
\hline $\mathbf{F}$ & $\begin{array}{c}108 \\
(4.31) \\
\end{array}$ & $\begin{array}{c}2220 \\
(23.12)\end{array}$ & $\begin{array}{c}2916 \\
(30.21)\end{array}$ & $\begin{array}{c}828 \\
(8.67) \\
\end{array}$ & $\begin{array}{c}375 \\
(3.93) \\
\end{array}$ & $\begin{array}{c}25 \\
(0.25) \\
\end{array}$ & $\begin{array}{c}30 \\
(0.31)\end{array}$ & $\begin{array}{c}36 \\
(0.37)\end{array}$ & $\begin{array}{c}45 \\
(0.46) \\
\end{array}$ & $\begin{array}{c}24 \\
(0.24) \\
\end{array}$ & $\begin{array}{c}21 \\
(0.21) \\
\end{array}$ & $\begin{array}{c}378 \\
(3.95) \\
\end{array}$ & $\begin{array}{c}856 \\
(8.96) \\
\end{array}$ & $\begin{array}{c}2899 \\
(30.06)\end{array}$ & $\begin{array}{c}2300 \\
(23.92)\end{array}$ & $\begin{array}{c}115 \\
(4.65)\end{array}$ \\
\hline $\mathbf{M}$ & $\begin{array}{c}157 \\
(6.26) \\
\end{array}$ & $\begin{array}{c}536 \\
(5.58)\end{array}$ & $\begin{array}{c}2066 \\
(21.4)\end{array}$ & $\begin{array}{c}262 \\
(2.74) \\
\end{array}$ & $\begin{array}{c}103 \\
(1.08)\end{array}$ & $\begin{array}{c}13 \\
(0.13) \\
\end{array}$ & $\begin{array}{c}15 \\
(0.15) \\
\end{array}$ & $\begin{array}{c}14 \\
(0.14)\end{array}$ & $\begin{array}{c}13 \\
(0.13) \\
\end{array}$ & $\begin{array}{c}12 \\
(0.12)\end{array}$ & $\begin{array}{c}12 \\
(0.12) \\
\end{array}$ & $\begin{array}{c}104 \\
(1.09) \\
\end{array}$ & $\begin{array}{c}234 \\
(2.45) \\
\end{array}$ & $\begin{array}{c}2124 \\
(22.03) \\
\end{array}$ & $\begin{array}{c}518 \\
(5.39)\end{array}$ & $\begin{array}{c}134 \\
(5.42) \\
\end{array}$ \\
\hline $\mathbf{P}$ & $\begin{array}{c}4 \\
(0.16) \\
\end{array}$ & $\begin{array}{c}5 \\
(0.05) \\
\end{array}$ & $\begin{array}{c}11 \\
(0.11) \\
\end{array}$ & $\begin{array}{c}6 \\
(0.06) \\
\end{array}$ & $\begin{array}{c}5 \\
(0.05) \\
\end{array}$ & $\begin{array}{c}4 \\
(0.04) \\
\end{array}$ & $\begin{array}{c}3 \\
(0.03) \\
\end{array}$ & $\begin{array}{c}4 \\
(0.04) \\
\end{array}$ & $\begin{array}{c}3 \\
(0.03) \\
\end{array}$ & $\begin{array}{c}3 \\
(0.03) \\
\end{array}$ & $\begin{array}{c}4 \\
(0.04) \\
\end{array}$ & $\begin{array}{c}6 \\
(0.06) \\
\end{array}$ & $\begin{array}{c}4 \\
(0.04) \\
\end{array}$ & $\begin{array}{c}10 \\
(0.1)\end{array}$ & $\begin{array}{c}5 \\
(0.05) \\
\end{array}$ & $\begin{array}{c}4 \\
(0.16) \\
\end{array}$ \\
\hline \multicolumn{17}{|c|}{ Adolescent (Female)* - 2010} \\
\hline $\mathbf{H}$ & $\begin{array}{c}178 \\
(97.8)\end{array}$ & $\begin{array}{c}1238 \\
(89.71)\end{array}$ & $\begin{array}{c}1394 \\
(67.67)\end{array}$ & $\begin{array}{c}2109 \\
(94.03)\end{array}$ & $\begin{array}{c}2080 \\
(89.54)\end{array}$ & $\begin{array}{c}2370 \\
(99)\end{array}$ & $\begin{array}{c}2402 \\
(95.89)\end{array}$ & $\begin{array}{c}2528 \\
(88.67)\end{array}$ & $\begin{array}{c}2519 \\
(88.39)\end{array}$ & $\begin{array}{l}2388 \\
(95.6)\end{array}$ & $\begin{array}{c}2356 \\
(99.03)\end{array}$ & $\begin{array}{c}2077 \\
(89.95)\end{array}$ & $\begin{array}{c}1923 \\
(93.08)\end{array}$ & $\begin{array}{c}1316 \\
(70.07)\end{array}$ & $\begin{array}{c}1158 \\
(88.87)\end{array}$ & $\begin{array}{c}215 \\
(83.66)\end{array}$ \\
\hline D & $\begin{array}{c}3 \\
(1.65) \\
\end{array}$ & $\begin{array}{c}91 \\
(6.59) \\
\end{array}$ & $\begin{array}{c}247 \\
(11.99) \\
\end{array}$ & $\begin{array}{c}52 \\
(2.32) \\
\end{array}$ & $\begin{array}{c}67 \\
(2.88) \\
\end{array}$ & $\begin{array}{c}14 \\
(0.58) \\
\end{array}$ & $\begin{array}{c}63 \\
(2.51) \\
\end{array}$ & $\begin{array}{c}156 \\
(5.47) \\
\end{array}$ & $\begin{array}{c}142 \\
(4.98) \\
\end{array}$ & $\begin{array}{c}62 \\
(2.48) \\
\end{array}$ & $\begin{array}{c}14 \\
(0.59) \\
\end{array}$ & $\begin{array}{c}72 \\
(3.12) \\
\end{array}$ & $\begin{array}{c}52 \\
(2.52) \\
\end{array}$ & $\begin{array}{c}200 \\
(10.65) \\
\end{array}$ & $\begin{array}{c}92 \\
(7.06)\end{array}$ & $\begin{array}{c}2 \\
(0.78) \\
\end{array}$ \\
\hline $\mathbf{F}$ & $\begin{array}{c}1 \\
(0.55) \\
\end{array}$ & $\begin{array}{c}48 \\
(3.48) \\
\end{array}$ & $\begin{array}{c}367 \\
(17.82) \\
\end{array}$ & $\begin{array}{c}52 \\
(2.32) \\
\end{array}$ & $\begin{array}{c}122 \\
(5.25) \\
\end{array}$ & $\begin{array}{c}7 \\
(0.29) \\
\end{array}$ & $\begin{array}{c}31 \\
(1.24) \\
\end{array}$ & $\begin{array}{c}155 \\
(5.44) \\
\end{array}$ & $\begin{array}{c}172 \\
(6.04)\end{array}$ & $\begin{array}{c}41 \\
(1.64)\end{array}$ & $\begin{array}{c}5 \\
(0.21) \\
\end{array}$ & $\begin{array}{c}106 \\
(4.59)\end{array}$ & $\begin{array}{c}68 \\
(3.29) \\
\end{array}$ & $\begin{array}{c}317 \\
(16.88)\end{array}$ & $\begin{array}{c}51 \\
(3.91) \\
\end{array}$ & $\begin{array}{c}2 \\
(0.78)\end{array}$ \\
\hline $\mathbf{M}$ & $\begin{array}{c}0 \\
(0)\end{array}$ & $\begin{array}{c}3 \\
(0.22)\end{array}$ & $\begin{array}{c}51 \\
(2.48)\end{array}$ & $\begin{array}{c}30 \\
(1.34)\end{array}$ & $\begin{array}{c}54 \\
(2.32) \\
\end{array}$ & $\begin{array}{c}3 \\
(0.13)\end{array}$ & $\begin{array}{c}8 \\
(0.32)\end{array}$ & $\begin{array}{c}10 \\
(0.35)\end{array}$ & $\begin{array}{c}12 \\
(0.42)\end{array}$ & $\begin{array}{c}6 \\
(0.24)\end{array}$ & $\begin{array}{c}3 \\
(0.13)\end{array}$ & $\begin{array}{c}54 \\
(2.34) \\
\end{array}$ & $\begin{array}{c}22 \\
(1.06)\end{array}$ & $\begin{array}{c}44 \\
(2.34)\end{array}$ & $\begin{array}{c}2 \\
(0.15)\end{array}$ & $\begin{array}{c}37 \\
(14.4) \\
\end{array}$ \\
\hline \multirow[t]{3}{*}{$\mathbf{P}$} & $\begin{array}{c}0 \\
(0) \\
\end{array}$ & $\begin{array}{c}0 \\
(0) \\
\end{array}$ & $\begin{array}{c}1 \\
(0.05)\end{array}$ & $\begin{array}{c}0 \\
(0) \\
\end{array}$ & $\begin{array}{c}0 \\
(0) \\
\end{array}$ & $\begin{array}{c}0 \\
(0) \\
\end{array}$ & $\begin{array}{c}1 \\
(0.04) \\
\end{array}$ & $\begin{array}{c}2 \\
(0.07) \\
\end{array}$ & $\begin{array}{c}5 \\
(0.18) \\
\end{array}$ & $\begin{array}{c}1 \\
(0.04)\end{array}$ & $\begin{array}{c}1 \\
(0.04) \\
\end{array}$ & $\begin{array}{c}0 \\
(0)\end{array}$ & $\begin{array}{c}1 \\
(0.05) \\
\end{array}$ & $\begin{array}{c}1 \\
(0.05) \\
\end{array}$ & $\begin{array}{c}0 \\
(0)\end{array}$ & $\begin{array}{c}1 \\
(0.39) \\
\end{array}$ \\
\hline & 18 & 17 & 16 & 15 & 14 & 13 & 12 & 11 & 21 & 22 & 23 & 24 & 25 & 26 & 27 & 28 \\
\hline & 48 & 47 & 46 & 45 & 44 & 43 & 42 & 41 & 31 & 32 & 33 & 34 & 35 & 36 & 37 & 38 \\
\hline $\mathbf{H}$ & $\begin{array}{c}211 \\
(88.66)\end{array}$ & $\begin{array}{c}969 \\
(84.19)\end{array}$ & $\begin{array}{c}1163 \\
(50.28)\end{array}$ & $\begin{array}{l}2372 \\
(91.3) \\
\end{array}$ & $\begin{array}{c}2631 \\
(94.71)\end{array}$ & $\begin{array}{c}2827 \\
(99.51)\end{array}$ & $\begin{array}{c}2846 \\
(99.51)\end{array}$ & $\begin{array}{c}2866 \\
(98.86)\end{array}$ & $\begin{array}{c}2869 \\
(98.9)\end{array}$ & $\begin{array}{c}2858 \\
(99.65)\end{array}$ & $\begin{array}{c}2838 \\
(99.33)\end{array}$ & $\begin{array}{c}2624 \\
(94.25)\end{array}$ & $\begin{array}{c}2399 \\
(92.45)\end{array}$ & $\begin{array}{c}1186 \\
(50.62)\end{array}$ & $\begin{array}{c}971 \\
(82.36)\end{array}$ & $\begin{array}{c}185 \\
(83.71) \\
\end{array}$ \\
\hline D & $\begin{array}{c}1 \\
(0.42)\end{array}$ & $\begin{array}{c}103 \\
(8.95)\end{array}$ & $\begin{array}{c}335 \\
(14.48)\end{array}$ & $\begin{array}{c}84 \\
(3.23) \\
\end{array}$ & $\begin{array}{c}31 \\
(1.12)\end{array}$ & $\begin{array}{c}10 \\
(0.35)\end{array}$ & $\begin{array}{c}7 \\
(0.24)\end{array}$ & $\begin{array}{c}22 \\
(0.76)\end{array}$ & $\begin{array}{c}17 \\
(0.591)\end{array}$ & $\begin{array}{c}6 \\
(0.21)\end{array}$ & $\begin{array}{c}12 \\
(0.42)\end{array}$ & $\begin{array}{c}35 \\
(1.26)\end{array}$ & $\begin{array}{c}64 \\
(2.47)\end{array}$ & $\begin{array}{c}339 \\
(14.47)\end{array}$ & $\begin{array}{c}120 \\
(10.18)\end{array}$ & $\begin{array}{c}1 \\
(0.45)\end{array}$ \\
\hline $\mathbf{F}$ & $\begin{array}{c}2 \\
(0.84) \\
\end{array}$ & $\begin{array}{c}61 \\
(5.3) \\
\end{array}$ & $\begin{array}{c}596 \\
(25.77) \\
\end{array}$ & $\begin{array}{c}99 \\
(3.81) \\
\end{array}$ & $\begin{array}{c}76 \\
(2.74) \\
\end{array}$ & $\begin{array}{c}4 \\
(0.14) \\
\end{array}$ & $\begin{array}{c}5 \\
(0.17) \\
\end{array}$ & $\begin{array}{c}10 \\
(0.34)\end{array}$ & $\begin{array}{c}12 \\
(0.41)\end{array}$ & $\begin{array}{c}2 \\
(0.07) \\
\end{array}$ & $\begin{array}{c}4 \\
(0.14) \\
\end{array}$ & $\begin{array}{c}82 \\
(2.95) \\
\end{array}$ & $\begin{array}{c}96 \\
(3.7) \\
\end{array}$ & $\begin{array}{c}577 \\
(24.63) \\
\end{array}$ & $\begin{array}{c}75 \\
(6.36) \\
\end{array}$ & $\begin{array}{c}2 \\
(0.9) \\
\end{array}$ \\
\hline $\mathbf{M}$ & $\begin{array}{c}24 \\
(10.08) \\
\end{array}$ & $\begin{array}{c}18 \\
(1.56) \\
\end{array}$ & $\begin{array}{c}218 \\
(9.42) \\
\end{array}$ & $\begin{array}{c}43 \\
(1.66) \\
\end{array}$ & $\begin{array}{c}39 \\
(1.4) \\
\end{array}$ & $\begin{array}{c}0 \\
(0) \\
\end{array}$ & $\begin{array}{c}2 \\
(0.07) \\
\end{array}$ & $\begin{array}{c}1 \\
(0.03) \\
\end{array}$ & $\begin{array}{c}3 \\
(0.1) \\
\end{array}$ & $\begin{array}{c}2 \\
(0.07) \\
\end{array}$ & $\begin{array}{c}3 \\
(0.11) \\
\end{array}$ & $\begin{array}{c}43 \\
(1.54) \\
\end{array}$ & $\begin{array}{c}36 \\
(1.39) \\
\end{array}$ & $\begin{array}{c}241 \\
(10.29) \\
\end{array}$ & $\begin{array}{c}13 \\
(1.1) \\
\end{array}$ & $\begin{array}{c}33 \\
(14.93) \\
\end{array}$ \\
\hline $\mathbf{P}$ & $\begin{array}{c}0 \\
(0) \\
\end{array}$ & $\begin{array}{c}0 \\
(0) \\
\end{array}$ & $\begin{array}{c}1 \\
(0.04) \\
\end{array}$ & $\begin{array}{c}0 \\
(0) \\
\end{array}$ & $\begin{array}{c}1 \\
(0.04) \\
\end{array}$ & $\begin{array}{c}0 \\
(0) \\
\end{array}$ & $\begin{array}{c}0 \\
(0) \\
\end{array}$ & $\begin{array}{c}0 \\
(0) \\
\end{array}$ & $\begin{array}{c}0 \\
(0)\end{array}$ & $\begin{array}{c}0 \\
(0) \\
\end{array}$ & $\begin{array}{c}0 \\
(0) \\
\end{array}$ & $\begin{array}{c}0 \\
(0)\end{array}$ & $\begin{array}{c}0 \\
(0)\end{array}$ & $\begin{array}{c}0 \\
(0)\end{array}$ & $\begin{array}{c}0 \\
(0)\end{array}$ & $\begin{array}{c}0 \\
(0)\end{array}$ \\
\hline
\end{tabular}

${ }^{*} \mathrm{p}<0.001$ Association between sample and codes. 
Table 3. The distribution of coincidence among homologous teeth according to dental condition and gender $(H=$ higid; $D=$ decayed; $M=$ missed; $F=$ filled; $P=$ prosthesis $)$

\begin{tabular}{|c|c|c|c|c|c|c|c|c|c|c|c|c|c|c|c|c|}
\hline \multicolumn{9}{|c|}{ Adolescent (Male) - 2003} & \multicolumn{8}{|c|}{ Adolescent (Female) - 2003} \\
\hline $\mathbf{H}$ & $\begin{array}{c}902 \\
(90.2) \\
\end{array}$ & $\begin{array}{c}3876 \\
(75.22) \\
\end{array}$ & $\begin{array}{c}2528 \\
(53.17)\end{array}$ & $\begin{array}{c}5234 \\
(91.89) \\
\end{array}$ & $\begin{array}{c}5289 \\
(91.79)\end{array}$ & $\begin{array}{c}6571 \\
(98.81)\end{array}$ & $\begin{array}{c}5519 \\
(91.03) \\
\end{array}$ & $\begin{array}{c}5300 \\
(84.62) \\
\end{array}$ & $\begin{array}{c}1207 \\
(86.71) \\
\end{array}$ & $\begin{array}{l}4919 \\
(69.2) \\
\end{array}$ & $\begin{array}{c}3355 \\
(50.05) \\
\end{array}$ & $\begin{array}{c}6968 \\
(90.29)\end{array}$ & $\begin{array}{c}7107 \\
(90.05) \\
\end{array}$ & $\begin{array}{c}9166 \\
(98.73) \\
\end{array}$ & $\begin{array}{c}7672 \\
(90.25) \\
\end{array}$ & $\begin{array}{c}7414 \\
(84.63) \\
\end{array}$ \\
\hline D & $\begin{array}{c}39 \\
(3.9) \\
\end{array}$ & $\begin{array}{c}735 \\
(14.26) \\
\end{array}$ & $\begin{array}{c}821 \\
(17.27) \\
\end{array}$ & $\begin{array}{c}153 \\
(2.69) \\
\end{array}$ & $\begin{array}{c}142 \\
(2.46) \\
\end{array}$ & $\begin{array}{c}35 \\
(0.53) \\
\end{array}$ & $\begin{array}{c}296 \\
(4.88) \\
\end{array}$ & $\begin{array}{c}521 \\
(8.32) \\
\end{array}$ & $\begin{array}{c}65 \\
(4.67) \\
\end{array}$ & $\begin{array}{c}1122 \\
(15.79)\end{array}$ & $\begin{array}{c}989 \\
(14.75) \\
\end{array}$ & $\begin{array}{c}231 \\
(2.99) \\
\end{array}$ & $\begin{array}{c}230 \\
(2.91) \\
\end{array}$ & $\begin{array}{c}48 \\
(0.52) \\
\end{array}$ & $\begin{array}{c}400 \\
(4.71)\end{array}$ & $\begin{array}{c}619 \\
(7.07) \\
\end{array}$ \\
\hline $\mathbf{F}$ & $\begin{array}{c}15 \\
(1.5)\end{array}$ & $\begin{array}{c}508 \\
(9.86) \\
\end{array}$ & $\begin{array}{c}1227 \\
(25.8) \\
\end{array}$ & $\begin{array}{c}264 \\
(4.63) \\
\end{array}$ & $\begin{array}{c}246 \\
(4.27) \\
\end{array}$ & $\begin{array}{c}25 \\
(0.38) \\
\end{array}$ & $\begin{array}{c}179 \\
(2.95) \\
\end{array}$ & $\begin{array}{c}346 \\
(5.52) \\
\end{array}$ & $\begin{array}{c}25 \\
(1.8) \\
\end{array}$ & $\begin{array}{l}995 \\
(14) \\
\end{array}$ & $\begin{array}{c}2007 \\
(29.94) \\
\end{array}$ & $\begin{array}{c}444 \\
(5.75) \\
\end{array}$ & $\begin{array}{c}428 \\
(5.42) \\
\end{array}$ & $\begin{array}{c}30 \\
(0.32) \\
\end{array}$ & $\begin{array}{c}283 \\
(3.33) \\
\end{array}$ & $\begin{array}{l}552 \\
(6.3) \\
\end{array}$ \\
\hline $\mathbf{M}$ & $\begin{array}{c}42 \\
(4.2) \\
\end{array}$ & $\begin{array}{c}31 \\
(0.6) \\
\end{array}$ & $\begin{array}{c}176 \\
(3.7) \\
\end{array}$ & $\begin{array}{c}42 \\
(0.74) \\
\end{array}$ & $\begin{array}{c}81 \\
(1.41) \\
\end{array}$ & $\begin{array}{c}16 \\
(0.24) \\
\end{array}$ & $\begin{array}{c}65 \\
(1.07) \\
\end{array}$ & $\begin{array}{c}88 \\
(1.41) \\
\end{array}$ & $\begin{array}{c}92 \\
(6.61) \\
\end{array}$ & $\begin{array}{c}69 \\
(0.97) \\
\end{array}$ & $\begin{array}{c}348 \\
(5.19) \\
\end{array}$ & $\begin{array}{c}71 \\
(0.92) \\
\end{array}$ & $\begin{array}{c}122 \\
(1.55)\end{array}$ & $\begin{array}{c}36 \\
(0.39) \\
\end{array}$ & $\begin{array}{c}143 \\
(1.68)\end{array}$ & $\begin{array}{c}164 \\
(1.87) \\
\end{array}$ \\
\hline \multirow[t]{3}{*}{$\mathbf{P}$} & $\begin{array}{c}2 \\
(0.2) \\
\end{array}$ & $\begin{array}{c}3 \\
(0.06) \\
\end{array}$ & $\begin{array}{c}3 \\
(0.06) \\
\end{array}$ & $\begin{array}{c}3 \\
(0.05)\end{array}$ & $\begin{array}{c}4 \\
(0.07) \\
\end{array}$ & $\begin{array}{c}3 \\
(0.05)\end{array}$ & $\begin{array}{c}4 \\
(0.07) \\
\end{array}$ & $\begin{array}{c}8 \\
(0.13) \\
\end{array}$ & $\begin{array}{c}3 \\
(0.22) \\
\end{array}$ & $\begin{array}{c}3 \\
(0.04) \\
\end{array}$ & $\begin{array}{c}4 \\
(0.06) \\
\end{array}$ & $\begin{array}{c}3 \\
(0.04) \\
\end{array}$ & $\begin{array}{c}5 \\
(0.06) \\
\end{array}$ & $\begin{array}{c}4 \\
(0.04) \\
\end{array}$ & $\begin{array}{c}3 \\
(0.04) \\
\end{array}$ & $\begin{array}{c}12 \\
(0.14)\end{array}$ \\
\hline & $18-28$ & $17-27$ & $16-26$ & $15-25$ & $14-24$ & $13-23$ & $12-22$ & $11-21$ & $18-28$ & $17-27$ & $16-26$ & $15-25$ & $14-24$ & $13-23$ & $12-22$ & $11-21$ \\
\hline & $38-48$ & $37-47$ & $36-46$ & $35-45$ & $34-44$ & $33-43$ & $32-42$ & $31-41$ & $38-48$ & $37-47$ & $36-46$ & $35-45$ & $34-44$ & $33-43$ & $32-32$ & $31-41$ \\
\hline $\mathbf{H}$ & $\begin{array}{c}1114 \\
(89.84) \\
\end{array}$ & $\begin{array}{c}2753 \\
(57.32) \\
\end{array}$ & $\begin{array}{c}1621 \\
(36.38) \\
\end{array}$ & $\begin{array}{c}5312 \\
(92.96) \\
\end{array}$ & $\begin{array}{c}6091 \\
(96.79) \\
\end{array}$ & $\begin{array}{c}6886 \\
(99.55) \\
\end{array}$ & $\begin{array}{c}6823 \\
(99.17) \\
\end{array}$ & $\begin{array}{c}6818 \\
(98.73) \\
\end{array}$ & $\begin{array}{c}1407 \\
(85.38) \\
\end{array}$ & $\begin{array}{c}3380 \\
(51.43) \\
\end{array}$ & $\begin{array}{c}1861 \\
(29.52) \\
\end{array}$ & $\begin{array}{c}7113 \\
(91.15) \\
\end{array}$ & $\begin{array}{c}8451 \\
(96.73) \\
\end{array}$ & $\begin{array}{c}9637 \\
(99.63) \\
\end{array}$ & $\begin{array}{c}9607 \\
(99.48) \\
\end{array}$ & $\begin{array}{c}9583 \\
(99.06) \\
\end{array}$ \\
\hline D & $\begin{array}{c}42 \\
(3.39) \\
\end{array}$ & $\begin{array}{c}1134 \\
(23.61) \\
\end{array}$ & $\begin{array}{c}821 \\
(18.42)\end{array}$ & $\begin{array}{c}139 \\
(2.43) \\
\end{array}$ & $\begin{array}{c}41 \\
(0.65) \\
\end{array}$ & $\begin{array}{c}15 \\
(0.22) \\
\end{array}$ & $\begin{array}{c}36 \\
(0.52) \\
\end{array}$ & $\begin{array}{c}60 \\
(0.87) \\
\end{array}$ & $\begin{array}{c}103 \\
(6.25) \\
\end{array}$ & $\begin{array}{c}1401 \\
(21.32)\end{array}$ & $\begin{array}{c}1060 \\
(16.81) \\
\end{array}$ & $\begin{array}{c}167 \\
(2.14) \\
\end{array}$ & $\begin{array}{c}46 \\
(0.53) \\
\end{array}$ & $\begin{array}{c}19 \\
(0.2) \\
\end{array}$ & $\begin{array}{c}30 \\
(0.31) \\
\end{array}$ & $\begin{array}{c}55 \\
(0.57) \\
\end{array}$ \\
\hline $\mathbf{F}$ & $\begin{array}{c}29 \\
(2.34) \\
\end{array}$ & $\begin{array}{c}811 \\
(16.89) \\
\end{array}$ & $\begin{array}{c}1324 \\
(29.71) \\
\end{array}$ & $\begin{array}{c}221 \\
(3.87)\end{array}$ & $\begin{array}{c}119 \\
(1.89) \\
\end{array}$ & $\begin{array}{c}6 \\
(0.09) \\
\end{array}$ & $\begin{array}{c}8 \\
(0.12) \\
\end{array}$ & $\begin{array}{c}18 \\
(0.26) \\
\end{array}$ & $\begin{array}{c}42 \\
(2.55) \\
\end{array}$ & $\begin{array}{c}1575 \\
(23.97)\end{array}$ & $\begin{array}{c}2085 \\
(33.07) \\
\end{array}$ & $\begin{array}{c}445 \\
(5.7) \\
\end{array}$ & $\begin{array}{c}186 \\
(2.13) \\
\end{array}$ & $\begin{array}{c}8 \\
(0.08) \\
\end{array}$ & $\begin{array}{c}10 \\
(0.1) \\
\end{array}$ & $\begin{array}{c}24 \\
(0.25) \\
\end{array}$ \\
\hline $\mathbf{M}$ & $\begin{array}{c}53 \\
(4.27) \\
\end{array}$ & $\begin{array}{c}102 \\
(2.12)\end{array}$ & $\begin{array}{c}687 \\
(15.42) \\
\end{array}$ & $\begin{array}{c}39 \\
(0.68)\end{array}$ & $\begin{array}{c}39 \\
(0.62) \\
\end{array}$ & $\begin{array}{c}7 \\
(0.1)\end{array}$ & $\begin{array}{c}10 \\
(0.15)\end{array}$ & $\begin{array}{c}7 \\
(0.1)\end{array}$ & $\begin{array}{c}93 \\
(5.64)\end{array}$ & $\begin{array}{c}213 \\
(3.24)\end{array}$ & $\begin{array}{c}1294 \\
(20.53) \\
\end{array}$ & $\begin{array}{c}75 \\
(0.96)\end{array}$ & $\begin{array}{c}49 \\
(0.56)\end{array}$ & $\begin{array}{c}5 \\
(0.05) \\
\end{array}$ & $\begin{array}{c}7 \\
(0.07)\end{array}$ & $\begin{array}{c}9 \\
(0.09)\end{array}$ \\
\hline $\mathbf{P}$ & $\begin{array}{c}2 \\
(0.16)\end{array}$ & $\begin{array}{c}3 \\
(0.06)\end{array}$ & $\begin{array}{c}3 \\
(0.07)\end{array}$ & $\begin{array}{c}3 \\
(0.05)\end{array}$ & $\begin{array}{c}3 \\
(0.05)\end{array}$ & $\begin{array}{c}3 \\
(0.04)\end{array}$ & $\begin{array}{c}3 \\
(0.04)\end{array}$ & $\begin{array}{c}3 \\
(0.04)\end{array}$ & $\begin{array}{c}3 \\
(0.18)\end{array}$ & $\begin{array}{c}3 \\
(0.05)\end{array}$ & $\begin{array}{c}4 \\
(0.06)\end{array}$ & $\begin{array}{c}4 \\
(0.05)\end{array}$ & $\begin{array}{c}5 \\
(0.06) \\
\end{array}$ & $\begin{array}{c}4 \\
(0.04) \\
\end{array}$ & $\begin{array}{c}3 \\
(0.03)\end{array}$ & $\begin{array}{c}3 \\
(0.03)\end{array}$ \\
\hline
\end{tabular}

\begin{tabular}{|c|c|c|c|c|c|c|c|c|c|c|c|c|c|c|c|c|}
\hline \multicolumn{9}{|c|}{ Adolescent (Male) - 2010} & \multicolumn{8}{|c|}{ Adolescent (Female) - 2010} \\
\hline $\mathbf{H}$ & $\begin{array}{c}317 \\
(85.68) \\
\end{array}$ & $\begin{array}{c}1693 \\
(87.95) \\
\end{array}$ & $\begin{array}{c}1187 \\
(71.08)\end{array}$ & $\begin{array}{c}1944 \\
(95.53)\end{array}$ & $\begin{array}{c}1958 \\
(94.45)\end{array}$ & $\begin{array}{c}2330 \\
(99.02) \\
\end{array}$ & $\begin{array}{c}2110 \\
(95.91) \\
\end{array}$ & $\begin{array}{c}1947 \\
(93.02) \\
\end{array}$ & $\begin{array}{c}353 \\
(83.25)\end{array}$ & $\begin{array}{c}1796 \\
(82.76)\end{array}$ & $\begin{array}{c}1377 \\
(69.58) \\
\end{array}$ & $\begin{array}{c}2272 \\
(93.73)\end{array}$ & $\begin{array}{c}2315 \\
(93.38) \\
\end{array}$ & $\begin{array}{c}2776 \\
(99.57) \\
\end{array}$ & $\begin{array}{c}2498 \\
(94.62) \\
\end{array}$ & $\begin{array}{c}2396 \\
(92.3)\end{array}$ \\
\hline D & $\begin{array}{c}11 \\
(2.97)\end{array}$ & $\begin{array}{c}107 \\
(5.56)\end{array}$ & $\begin{array}{c}166 \\
(9.94)\end{array}$ & $\begin{array}{c}40 \\
(1.97)\end{array}$ & $\begin{array}{c}31 \\
(1.5) \\
\end{array}$ & $\begin{array}{c}14 \\
(0.59)\end{array}$ & $\begin{array}{c}55 \\
(2.5) \\
\end{array}$ & $\begin{array}{c}86 \\
(4.11) \\
\end{array}$ & $\begin{array}{c}10 \\
(2.367) \\
\end{array}$ & $\begin{array}{c}162 \\
(7.47)\end{array}$ & $\begin{array}{c}177 \\
(8.94)\end{array}$ & $\begin{array}{c}34 \\
(1.4) \\
\end{array}$ & $\begin{array}{c}34 \\
(1.37) \\
\end{array}$ & $\begin{array}{c}5 \\
(0.18) \\
\end{array}$ & $\begin{array}{c}72 \\
(2.73) \\
\end{array}$ & $\begin{array}{c}92 \\
(3.54) \\
\end{array}$ \\
\hline $\mathbf{F}$ & $\begin{array}{c}3 \\
(0.81) \\
\end{array}$ & $\begin{array}{c}119 \\
(6.18)\end{array}$ & $\begin{array}{c}291 \\
(17.43) \\
\end{array}$ & $\begin{array}{c}38 \\
(1.87) \\
\end{array}$ & $\begin{array}{c}51 \\
(2.46)\end{array}$ & $\begin{array}{c}7 \\
(0.3)\end{array}$ & $\begin{array}{c}25 \\
(1.14)\end{array}$ & $\begin{array}{c}53 \\
(2.53) \\
\end{array}$ & $\begin{array}{c}4 \\
(0.94) \\
\end{array}$ & $\begin{array}{c}204 \\
(9.4)\end{array}$ & $\begin{array}{c}381 \\
(19.25) \\
\end{array}$ & $\begin{array}{c}99 \\
(4.08)\end{array}$ & $\begin{array}{c}79 \\
(3.19)\end{array}$ & $\begin{array}{c}5 \\
(0.18) \\
\end{array}$ & $\begin{array}{c}59 \\
(2.23) \\
\end{array}$ & $\begin{array}{c}100 \\
(3.85)\end{array}$ \\
\hline $\mathbf{M}$ & $\begin{array}{c}39 \\
(10.54) \\
\end{array}$ & $\begin{array}{c}6 \\
(0.31) \\
\end{array}$ & $\begin{array}{c}26 \\
(1.56)\end{array}$ & $\begin{array}{c}12 \\
(0.64)\end{array}$ & $\begin{array}{c}33 \\
(1.59)\end{array}$ & $\begin{array}{c}2 \\
(0.08) \\
\end{array}$ & $\begin{array}{c}10 \\
(0.45)\end{array}$ & $\begin{array}{c}7 \\
(0.33) \\
\end{array}$ & $\begin{array}{c}57 \\
(13.44) \\
\end{array}$ & $\begin{array}{c}8 \\
(0.37) \\
\end{array}$ & $\begin{array}{c}44 \\
(2.22) \\
\end{array}$ & $\begin{array}{c}19 \\
(0.78)\end{array}$ & $\begin{array}{c}51 \\
(2.06)\end{array}$ & $\begin{array}{c}2 \\
(0.07)\end{array}$ & $\begin{array}{c}10 \\
(0.38)\end{array}$ & $\begin{array}{c}7 \\
(0.27)\end{array}$ \\
\hline \multirow[t]{3}{*}{$\mathbf{P}$} & $\begin{array}{c}0 \\
(0) \\
\end{array}$ & $\begin{array}{c}0 \\
(0)\end{array}$ & $\begin{array}{c}0 \\
(0)\end{array}$ & $\begin{array}{c}0 \\
(0)\end{array}$ & $\begin{array}{c}0 \\
(0)\end{array}$ & $\begin{array}{c}0 \\
(0)\end{array}$ & $\begin{array}{c}0 \\
(0)\end{array}$ & $\begin{array}{c}0 \\
(0)\end{array}$ & $\begin{array}{c}0 \\
(0) \\
\end{array}$ & $\begin{array}{c}0 \\
(0)\end{array}$ & $\begin{array}{c}0 \\
(0)\end{array}$ & $\begin{array}{c}0 \\
(0)\end{array}$ & $\begin{array}{c}0 \\
(0)\end{array}$ & $\begin{array}{c}0 \\
(0)\end{array}$ & $\begin{array}{c}1 \\
(0.04)\end{array}$ & $\begin{array}{c}1 \\
(0.04)\end{array}$ \\
\hline & $18-28$ & $17-27$ & $16-26$ & $15-25$ & $14-24$ & $13-23$ & $12-22$ & $11-21$ & $18-28$ & $17-27$ & $16-26$ & $15-25$ & $14-24$ & $13-23$ & $12-22$ & $11-21$ \\
\hline & $38-48$ & $37-47$ & $36-46$ & $35-45$ & $34-44$ & $33-43$ & $32-42$ & $31-41$ & $38-48$ & $37-47$ & $36-46$ & $35-45$ & $34-44$ & $33-43$ & $32-42$ & $31-41$ \\
\hline $\mathbf{H}$ & $\begin{array}{c}424 \\
(86.71) \\
\end{array}$ & $\begin{array}{c}1308 \\
(73.65) \\
\end{array}$ & $\begin{array}{c}935 \\
(57.82) \\
\end{array}$ & $\begin{array}{c}2051 \\
(96.88) \\
\end{array}$ & $\begin{array}{c}2183 \\
(97.37) \\
\end{array}$ & $\begin{array}{c}2401 \\
(99.67) \\
\end{array}$ & $\begin{array}{l}2393 \\
(99.5) \\
\end{array}$ & $\begin{array}{c}2371 \\
(99.54) \\
\end{array}$ & $\begin{array}{c}434 \\
(84.44) \\
\end{array}$ & $\begin{array}{c}1372 \\
(67.52) \\
\end{array}$ & $\begin{array}{c}1026 \\
(54.17) \\
\end{array}$ & $\begin{array}{c}2349 \\
(95.53) \\
\end{array}$ & $\begin{array}{c}2608 \\
(97.46) \\
\end{array}$ & $\begin{array}{c}2865 \\
(99.79) \\
\end{array}$ & $\begin{array}{c}2868 \\
(99.65) \\
\end{array}$ & $\begin{array}{c}2837 \\
(99.58) \\
\end{array}$ \\
\hline D & $\begin{array}{c}20 \\
(4.09)\end{array}$ & $\begin{array}{c}245 \\
(13.8)\end{array}$ & $\begin{array}{c}188 \\
(11.63)\end{array}$ & $\begin{array}{c}18 \\
(0.85)\end{array}$ & $\begin{array}{c}13 \\
(0.58)\end{array}$ & $\begin{array}{c}5 \\
(0.21)\end{array}$ & $\begin{array}{c}6 \\
(0.25)\end{array}$ & $\begin{array}{c}5 \\
(0.21)\end{array}$ & $\begin{array}{c}13 \\
(2.53)\end{array}$ & $\begin{array}{c}283 \\
(13.93)\end{array}$ & $\begin{array}{c}212 \\
(11.19)\end{array}$ & $\begin{array}{c}22 \\
(0.89)\end{array}$ & $\begin{array}{c}8 \\
(0.3)\end{array}$ & $\begin{array}{c}2 \\
(0.07)\end{array}$ & $\begin{array}{c}5 \\
(0.17)\end{array}$ & $\begin{array}{c}6 \\
(0.21)\end{array}$ \\
\hline $\mathbf{F}$ & $\begin{array}{c}3 \\
(0.61)\end{array}$ & $\begin{array}{c}208 \\
(11.71) \\
\end{array}$ & $\begin{array}{c}403 \\
(24.92) \\
\end{array}$ & $\begin{array}{c}37 \\
(1.75)\end{array}$ & $\begin{array}{c}25 \\
(1.12)\end{array}$ & $\begin{array}{c}2 \\
(0.08)\end{array}$ & $\begin{array}{c}4 \\
(0.17)\end{array}$ & $\begin{array}{c}4 \\
(0.17)\end{array}$ & $\begin{array}{c}11 \\
(2.14)\end{array}$ & $\begin{array}{c}348 \\
(17.13)\end{array}$ & $\begin{array}{c}486 \\
(25.66) \\
\end{array}$ & $\begin{array}{c}70 \\
(2.85)\end{array}$ & $\begin{array}{c}29 \\
(1.08)\end{array}$ & $\begin{array}{c}4 \\
(0.14)\end{array}$ & $\begin{array}{c}5 \\
(0.17)\end{array}$ & $\begin{array}{c}5 \\
(0.18)\end{array}$ \\
\hline $\mathbf{M}$ & $\begin{array}{c}42 \\
(8.59) \\
\end{array}$ & $\begin{array}{c}15 \\
(0.84) \\
\end{array}$ & $\begin{array}{c}91 \\
(5.63) \\
\end{array}$ & $\begin{array}{c}11 \\
(0.52) \\
\end{array}$ & $\begin{array}{c}21 \\
(0.94) \\
\end{array}$ & $\begin{array}{c}1 \\
(0.04) \\
\end{array}$ & $\begin{array}{c}2 \\
(0.08) \\
\end{array}$ & $\begin{array}{c}2 \\
(0.08) \\
\end{array}$ & $\begin{array}{c}56 \\
(10.89) \\
\end{array}$ & $\begin{array}{c}29 \\
(1.43) \\
\end{array}$ & $\begin{array}{c}170 \\
(8.98) \\
\end{array}$ & $\begin{array}{c}18 \\
(0.73) \\
\end{array}$ & $\begin{array}{c}31 \\
(1.16) \\
\end{array}$ & $\begin{array}{c}0 \\
(0) \\
\end{array}$ & $\begin{array}{c}0 \\
(0) \\
\end{array}$ & $\begin{array}{c}1 \\
(0.04) \\
\end{array}$ \\
\hline $\mathbf{P}$ & $\begin{array}{c}0 \\
(0)\end{array}$ & $\begin{array}{c}0 \\
(0)\end{array}$ & $\begin{array}{c}0 \\
(0)\end{array}$ & $\begin{array}{c}0 \\
(0)\end{array}$ & $\begin{array}{c}0 \\
(0)\end{array}$ & $\begin{array}{c}0 \\
(0)\end{array}$ & $\begin{array}{c}0 \\
(0)\end{array}$ & $\begin{array}{c}0 \\
(0)\end{array}$ & $\begin{array}{c}0 \\
(0)\end{array}$ & $\begin{array}{c}0 \\
(0)\end{array}$ & $\begin{array}{c}0 \\
(0)\end{array}$ & $\begin{array}{c}0 \\
(0)\end{array}$ & $\begin{array}{c}0 \\
(0)\end{array}$ & $\begin{array}{c}0 \\
(0)\end{array}$ & $\begin{array}{c}0 \\
(0)\end{array}$ & $\begin{array}{c}0 \\
(0)\end{array}$ \\
\hline
\end{tabular}


Table 4. The distribution of ordinary and extraordinary findings according to dental condition, age group and gender $(H=$ higid; $D=$ decayed; $M=$ missed; $F=$ filled; $P=$ prosthesis)

\begin{tabular}{|c|c|c|c|c|c|c|c|c|c|c|c|c|c|c|c|c|}
\hline \multicolumn{17}{|c|}{ Adolescent - 2003} \\
\hline a & $\mathrm{H}$ & HDF & $\mathrm{HDF}$ & $\mathrm{H}$ & $\mathrm{H}$ & $\mathrm{H}$ & $\mathrm{H}$ & $\mathrm{HD}$ & $\mathrm{HD}$ & $\mathrm{H}$ & $\mathrm{H}$ & $\mathrm{H}$ & $\mathrm{H}$ & $\mathrm{HDF}$ & $\mathrm{HDF}$ & $\mathrm{H}$ \\
\hline $\mathbf{b}$ & DMFP & MP & MP & DMFP & DMFP & DMFP & DMFP & MFP & MFP & DMFP & DMFP & DMFP & DMFP & MP & MP & DMFP \\
\hline c & $\mathrm{H}$ & HDF & $\mathrm{HDF}$ & $\mathrm{H}$ & $\mathrm{H}$ & $\mathrm{H}$ & $\mathrm{H}$ & $\mathrm{H}$ & $\mathrm{H}$ & $\mathrm{H}$ & $\mathrm{H}$ & $\mathrm{H}$ & $\mathrm{H}$ & $\mathrm{HDF}$ & $\mathrm{HDF}$ & $\mathrm{H}$ \\
\hline \multirow[t]{3}{*}{ d } & DMFP & MP & MP & DMFP & DMFP & DMFP & DMFP & DMFP & DMFP & DMFP & DMFP & DMFP & DMFP & MP & MP & DMFP \\
\hline & 18 & 17 & 16 & 15 & 14 & 13 & 12 & 11 & 21 & 22 & 23 & 24 & 25 & 26 & 27 & 28 \\
\hline & 48 & 47 & 46 & 45 & 44 & 43 & 42 & 41 & 31 & 32 & 33 & 34 & 35 & 36 & 37 & 38 \\
\hline a & $\mathrm{H}$ & HDF & HDFM & $\mathrm{H}$ & $\mathrm{H}$ & $\mathrm{H}$ & $\mathrm{H}$ & $\mathrm{HD}$ & $\mathrm{HD}$ & $\mathrm{H}$ & $\mathrm{H}$ & $\mathrm{H}$ & $\mathrm{H}$ & HDF & HDFM & $\mathrm{H}$ \\
\hline $\mathbf{b}$ & DMFP & MP & $\mathrm{P}$ & DMFP & DMFP & DMFP & DMFP & MFP & MFP & DMFP & DMFP & DMFP & DMFP & MP & $\mathrm{P}$ & DMFP \\
\hline c & $\mathrm{H}$ & HDF & HDFM & $\mathrm{H}$ & $\mathrm{H}$ & $\mathrm{H}$ & $\mathrm{H}$ & $\mathrm{H}$ & $\mathrm{H}$ & $\mathrm{H}$ & $\mathrm{H}$ & $\mathrm{H}$ & $\mathrm{H}$ & HDFM & HDF & $\mathrm{H}$ \\
\hline d & DMFP & MP & $\mathrm{P}$ & DMFP & DMFP & DMFP & DMFP & DMFP & DMFP & DMFP & DMFP & DMFP & DMFP & $\mathrm{P}$ & MP & DMFP \\
\hline
\end{tabular}

\begin{tabular}{|c|c|c|c|c|c|c|c|c|c|c|c|c|c|c|c|c|}
\hline \multicolumn{17}{|c|}{ Adolescent - 2010} \\
\hline a & $\mathrm{H}$ & $\mathrm{H}$ & HDF & $\mathrm{H}$ & $\mathrm{H}$ & $\mathrm{H}$ & $\mathrm{H}$ & $\mathrm{H}$ & $\mathrm{H}$ & $\mathrm{H}$ & $\mathrm{H}$ & $\mathrm{H}$ & $\mathrm{H}$ & HDF & $\mathrm{H}$ & $\mathrm{H}$ \\
\hline b & DMFP & DMFP & MP & DMFP & DMFP & DMFP & DMFP & DMFP & DMFP & DMFP & DMFP & DMFP & DMFP & MP & DMFP & DMFP \\
\hline c & $\mathrm{H}$ & $\mathrm{H}$ & HDF & $\mathrm{H}$ & $\mathrm{H}$ & $\mathrm{H}$ & $\mathrm{H}$ & $\mathrm{H}$ & $\mathrm{H}$ & $\mathrm{H}$ & $\mathrm{H}$ & $\mathrm{H}$ & $\mathrm{H}$ & $\mathrm{HDF}$ & $\mathrm{H}$ & $\mathrm{HM}$ \\
\hline \multirow[t]{3}{*}{ d } & DMFP & DMFP & MP & DMFP & DMFP & DMFP & DMFP & DMFP & DMFP & DMFP & DMFP & DMFP & DMFP & MP & DMFP & DMFP \\
\hline & 18 & 17 & 16 & 15 & 14 & 13 & 12 & 11 & 21 & 22 & 23 & 24 & 25 & 26 & 27 & 28 \\
\hline & 48 & 47 & 46 & 45 & 44 & 43 & 42 & 41 & 31 & 32 & 33 & 34 & 35 & 36 & 37 & 38 \\
\hline a & $\mathrm{H}$ & $\mathrm{H}$ & HDF & $\mathrm{H}$ & $\mathrm{H}$ & $\mathrm{H}$ & $\mathrm{H}$ & $\mathrm{H}$ & $\mathrm{H}$ & $\mathrm{H}$ & $\mathrm{H}$ & $\mathrm{H}$ & $\mathrm{H}$ & $\mathrm{HDF}$ & $\mathrm{H}$ & $\mathrm{H}$ \\
\hline $\mathbf{b}$ & DMFP & DMFP & MP & DMFP & DMFP & DMFP & DMFP & DMFP & DMFP & DMFP & DMFP & DMFP & DMFP & MP & DMFP & DMFP \\
\hline c & HM & $\mathrm{H}$ & HDF & $\mathrm{H}$ & $\mathrm{H}$ & $\mathrm{H}$ & $\mathrm{H}$ & $\mathrm{H}$ & $\mathrm{H}$ & $\mathrm{H}$ & $\mathrm{H}$ & $\mathrm{H}$ & $\mathrm{H}$ & HDFM & $\mathrm{HD}$ & HM \\
\hline d & DFP & DMFP & MP & DMFP & DMFP & DMFP & DMFP & DMFP & DMFP & DMFP & DMFP & DMFP & DMFP & $\mathrm{P}$ & FMP & DFP \\
\hline
\end{tabular}


Table 5. The distribution of dental pattern diversity according to age group, gender and region

${ }^{e}$ Northern Region. ${ }^{f}$ Northeastern Region. ${ }^{g}$ Southeastern Region. ${ }^{b}$ Southern Region. ${ }^{i}$ Central-Western Region.

\begin{tabular}{|c|c|c|c|c|c|c|c|c|c|c|}
\hline \multicolumn{6}{|c|}{ Adolescent (Male) - 2003} & \multicolumn{5}{|c|}{ Adolescent (Female) - 2003} \\
\hline & $\mathrm{N}$ & $\begin{array}{l}\text { Pairwise } \\
\text { Comparation }\end{array}$ & Matches & $\begin{array}{l}\text { Total Diversity } \\
\text { Estimate }\end{array}$ & $\begin{array}{c}\text { Conditional } \\
\text { Diversity } \\
\text { Estimate }\end{array}$ & $\mathrm{N}$ & $\begin{array}{c}\text { Pairwise } \\
\text { Comparation }\end{array}$ & Matches & $\begin{array}{c}\text { Total } \\
\text { Diversity } \\
\text { Estimate }\end{array}$ & $\begin{array}{c}\text { Conditional } \\
\text { Diversity } \\
\text { Estimate }\end{array}$ \\
\hline $28^{a}$ & 7,015 & $24,601,605$ & $2,524,681$ & $0.897^{\mathrm{d}}$ & $0.997^{\mathrm{d}}$ & 9,818 & $48,191,653$ & $3,362,086$ & $0.930^{\mathrm{d}}$ & $0.981^{\mathrm{d}}$ \\
\hline $14 u^{b}$ & 7,015 & $24,601,605$ & $6,128,035$ & $0.751^{d}$ & $0.971^{\mathrm{d}}$ & 9,818 & $48,191,653$ & $9,971,663$ & $0.793^{d}$ & $0.973^{\mathrm{d}}$ \\
\hline $141^{\mathrm{c}}$ & 7,015 & $24,601,605$ & $4,196,894$ & $0.829^{\mathrm{d}}$ & $0.984^{d}$ & 9,818 & $48,191,653$ & $5,781,320$ & $0.880^{\mathrm{d}}$ & $0.984^{d}$ \\
\hline \multicolumn{6}{|c|}{ Adolescent (Male) - 2010} & \multicolumn{5}{|c|}{ Adolescent (Female) - 2010} \\
\hline & $\mathrm{N}$ & $\begin{array}{l}\text { Pairwise } \\
\text { Comparation }\end{array}$ & Matches & $\begin{array}{l}\text { Total Diversity } \\
\text { Estimate }\end{array}$ & $\begin{array}{l}\text { Conditional } \\
\text { Diversity } \\
\text { Estimate }\end{array}$ & $\mathrm{N}$ & $\begin{array}{c}\text { Pairwise } \\
\text { Comparation }\end{array}$ & Matches & $\begin{array}{c}\text { Total } \\
\text { Diversity } \\
\text { Estimate }\end{array}$ & $\begin{array}{c}\text { Conditional } \\
\text { Diversity } \\
\text { Estimate }\end{array}$ \\
\hline $28^{a}$ & 2,497 & $3,116,256$ & 215,678 & $0.931^{\mathrm{d}}$ & $0.98^{\mathrm{d}}$ & 2,948 & $4,343,878$ & 312228 & $0.928^{\mathrm{d}}$ & $0.971^{\mathrm{d}}$ \\
\hline $14 u^{b}$ & 2,497 & $3,116,256$ & 345,436 & $0.889^{\mathrm{d}}$ & $0.954^{\mathrm{d}}$ & 2,948 & $4,343,878$ & 399732 & $0.908^{\mathrm{d}}$ & $0.934^{\mathrm{d}}$ \\
\hline $141^{\mathrm{c}}$ & 2,497 & $3,116,256$ & 556,766 & $0.821^{\mathrm{d}}$ & $0.931^{\mathrm{d}}$ & 2,948 & $4,343,878$ & 476543 & $0.89^{\mathrm{d}}$ & $0.911^{\mathrm{d}}$ \\
\hline \multicolumn{6}{|c|}{ Adolescent (Male) 2010 - by Brazilian region - $28^{a}$} & \multicolumn{5}{|c|}{ Adolescent (Female) 2010 - by Brazilian region - 28 } \\
\hline & $\mathrm{N}$ & $\begin{array}{c}\text { Pairwise } \\
\text { Comparation }\end{array}$ & Matches & $\begin{array}{l}\text { Total Diversity } \\
\text { Estimate }\end{array}$ & $\begin{array}{c}\text { Conditional } \\
\text { Diversity } \\
\text { Estimate }\end{array}$ & $\mathrm{N}$ & $\begin{array}{c}\text { Pairwise } \\
\text { Comparation }\end{array}$ & Matches & $\begin{array}{c}\text { Total } \\
\text { Diversity } \\
\text { Estimate }\end{array}$ & $\begin{array}{c}\text { Conditional } \\
\text { Diversity } \\
\text { Estimate }\end{array}$ \\
\hline $1^{\mathrm{e}}$ & 597 & 177,906 & 7,868 & $0.956^{\mathrm{d}}$ & $0.981^{\mathrm{d}}$ & 770 & 296,065 & 17,341 & $0.941^{\mathrm{d}}$ & $0.951^{\mathrm{d}}$ \\
\hline $2^{\mathrm{f}}$ & 661 & 218,13 & 8,324 & $0.962^{\mathrm{d}}$ & $0.976^{\mathrm{d}}$ & 777 & 301,476 & 24,379 & $0.919^{d}$ & $0.932^{\mathrm{d}}$ \\
\hline $3^{\mathrm{g}}$ & 426 & 90,525 & 15,435 & $0.829^{d}$ & $0.991^{\mathrm{d}}$ & 487 & 118,341 & 18,765 & $0.841^{d}$ & $0.947^{\mathrm{d}}$ \\
\hline $4^{\mathrm{h}}$ & 397 & 78,606 & 17,822 & $0.773^{d}$ & $0.985^{d}$ & 421 & 88,41 & 16,543 & $0.813^{\mathrm{d}}$ & $0.933^{\mathrm{d}}$ \\
\hline $5^{i}$ & 416 & 86,32 & 9,345 & $0.892^{\mathrm{d}}$ & $0.983^{d}$ & 493 & 121,278 & 9,543 & $0.921^{d}$ & $0.971^{\mathrm{d}}$ \\
\hline
\end{tabular}

$\mathrm{p}>0.05$.

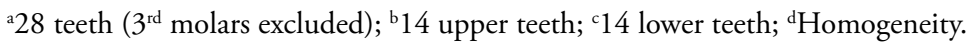

\section{REFERENCES}

1. Sweet D. Forensic dental identification. Forensic Sci Int. 2010;201(1-3):3-4.

2. Hill AJ, Lain R, Hewson I. Preservation of dental evidence following exposure to high temperatures. Forensic Sci Int. 2011;205(1-3):40-3.

3. Lake AW, James H, Berketa JW. Disaster victim identification: quality management from an odontology perspective. Forensic Sci Med Pathol. 2012;8(2):157-63.

4. Adams BJ. Establishing personal identification based on specific patterns of missing, filled, and unrestored teeth. J Forensic Sci. 2003;48(3):487-96.

5. Adams BJ. The diversity of adult dental patterns in the United States and the implications for personal identification. J Forensic Sci. 2003;48(3):497-503.

6. Martin-de-Las-Heras S, Valenzuela A, Luna Jde D, Bravo M. The utility of dental patterns in forensic dentistry. Forensic Sci Int. 2010;195(1-3):166.e1-5.

7. Biazevic MG, de Almeida NH, Crosato E, Michel-Crosato E. Diversity of dental patterns: application on different ages using the Brazilian National Oral Health Survey. Forensic Sci Int. 2011;207(1-3):240.e1-9.

8. Kumar A, Ghosh S, Logani A. Occurrence of diversity in dental pattern and their role in identification in Indian population: An orthopantomogram based pilot study. J Forensic Dent Sci. 2014;6(1):42-5.

9. Brasil. Projeto SB Brasil - 2003: Condições da saúde bucal da população brasileira 2002-2003 - Resultados principais. Brasília: Ministério da Saúde, 2004.

10. Brasil. Projeto SB Brasil - 2010: Pesquisa Nacional de Saúde Bucal Resultados principais. Brasília: Ministério da Saúde, 2011.
11. Roncalli AG. National oral health survey in 2010 shows a major decrease in dental caries in Brazil. Cad Saude Publica 2011;27(1):4-5. English, Portuguese.

12. Kasiulevicious V, Sapoka V, Filipaviciute R. Sample size calculation in epidemiological studies. Gerontologija 2006;7:225-231.

13. Sheiham A, Alexander D, Cohen L, Marinho V, Moysés S, Petersen PE, et al. Global oral health inequalities: task group-implementation and delivery of oral health strategies. Adv Dent Res. 2011;23(2):259-67.

14. Lee SS, Choi JH, Yoon CL, Kim CY, Shin KJ. The diversity of dental patterns in the orthopantomography and its significance in human identification. J Forensic Sci. 2004;49(4):784-6.

15. Pucca Junior GA, Lucena EH, Cawahisa PT. Financing national policy on oral health in Brazil in the context of the Unified Health System. Braz Oral Res. 2010;24(Suppl 1):26-32.

16. Brkic H, Strinovic D, Kubat M, Petrovecki V. Odontological identification of human remains from mass graves in Croatia. Int J Legal Med. 2000;114(1-2):19-22.

17. Muthusubramanian M, Limson KS, Julian R. Analysis of rugae in burn victims and cadavers to simulate rugae identification in cases of incineration and decomposition. J Forensic Odontostomatol. 2005;23(1):26-9.

18. Martínez-Chicón J, Valenzuela A. Usefulness of Forensic Dental Symbols@ and Dental Encoder $($ database in forensic odontology. J Forensic Sci. 2012;57(1):206-11.

19. Blau S, Hill A, Briggs CA, Cordner SM. Missing persons-missing data: the need to collect antemortem dental records of missing persons. J Forensic Sci. 2006;51(2):386-9. 
Conference proceedings

\section{CROATIAN DAYS OF PAEDIATRIC DENTISTRY 2014, CROATIAN SOCIETY FOR PAEDIATRIC AND PREVENTIVE DENTISTRY, ZAGREB, DECEMBER $5^{\text {th }}-6^{\text {th }}, 2014$}

\section{ENDODONTIC TREATMENT AS PREVENTION OF PREMATURE LOSS OF PRIMARY TEETH}

Danko Bakarčić *

* Department of Paediatric Dentistry, School of Medicine, University of Rijeka, Clinical Hospital Center Rijeka, Rijeka, Croatia

\section{Corresponding Author:}

Danko Bakarčić

Department of Paediatric Dentistry, School of Medicine, University of Rijeka, Clinical Hospital Center Rijeka,

Rijeka, Croatia

e-mail: danko.bakarcic@medri.uniri.hr

\section{Abstract}

Premature tooth loss is defined as tooth loss before the time of its natural exfoliation. In the primary dentition it causes serious complications, especially if a tendency towards orthodontic anomalies exists. Orthodontic anomalies could significantly impair the growth and development of the upper and lower jaw with the usual consequence of a long and expensive orthodontic treatment.

This lecture will describe the endodontic techniques that can prevent early loss of primary teeth until their natural replacement, in case of pulp infection.

Primary tooth is still the best space maintainer until the moment of replacement with permanent successor.

\section{THE ROLE OF DENTISTS IN DETECTING CELIAC DISEASE IN CHILDREN}

Ivana Čuković - Bagić *

* Department of Paediatric Dentistry, School of Dental Medicine, University of Zagreb, Zagreb, Croatia

Corresponding Author:

Ivana Čuković - Bagić

Department of Paediatric Dentistry, School of Dental

Medicine,

University of Zagreb,

Zagreb, Croatia

e-mail: bagic@sfzg.hr

\begin{abstract}
Celiac disease is a chronic immune disorder caused by intolerance to gluten in the diet, which occurs in persons with a genetic predisposition and can appear at any age. Diagnosis of the disease is carried out by serological testing and histopathological analysis of the small intestine mucosa. As symptoms are not often present in gastrointestinal tract, and serologic tests can be falsely negative, the confirmation of diagnosis is sometimes very prolonged. Since celiac disease causes disorders of intestinal absorption, and therefore the malabsorption of essential minerals and nutrients, it is expected that changes may occur in the structure of the enamel and therefore visible even in children. It is very important to diagnose the disease before the development of apparent clinical complications. This is possible by intensive and precise exploring of the symptoms on other (extraintestinal) organ systems that may help in the early identification of the disease. So far, several studies demonstrate that in patients with celiac disease a variety of oral lesions (changes of tooth enamel, recurrent aphthous stomatitis and atrophic glossitis) may be found, but of all the above, it is assumed that changes in the enamel may be the most important for celiac disease. This lecture will show, therefore, that noncarious lesions can indicate celiac disease and that the dentist could identify and accelerate the diagnosis of this life-long and incurable disease.
\end{abstract}

